\title{
Transport and continuity equations with (very) rough noise
}

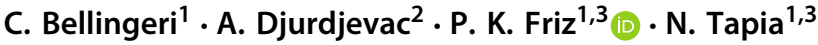

Received: 5 June 2020 / Accepted: 16 May 2021 / Published online: 21 June 2021

(C) The Author(s) 2021

\begin{abstract}
Existence and uniqueness for rough flows, transport and continuity equations driven by general geometric rough paths are established.
\end{abstract}

Keywords Rough transport equation - Rough continuity equation · First order rough partial differential equations

Mathematics Subject Classifications 35R60 - 60L20 - 60L50 · 60H15

\section{Contents}

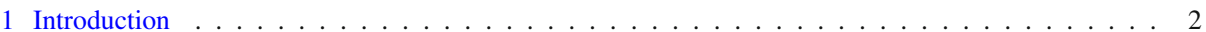

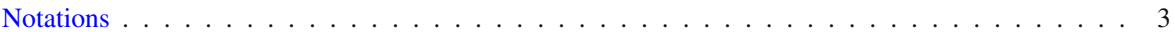

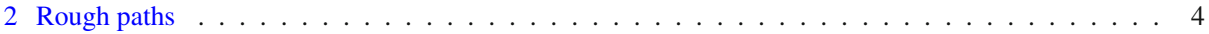

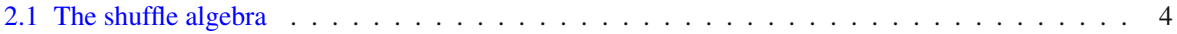

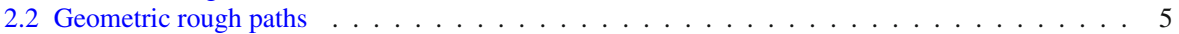

2.3 Controlled rough paths and rough integrals . . . . . . . . . . . . . . . . . . . 6

3 Rough differential equations . . . . . . . . . . . . . . . . . . . . . . . . 11

3.1 Differentiability of the flow . . . . . . . . . . . . . . . 13

3.2 Itô's formula for RDEs . . . . . . . . . . . . . . . . . . . . . . . . . . . . . . . . . . . 16

4 Rough transport and continuity . . . . . . . . . . . . . . . . . . . . . . . 19

4.1 Rough transport equation . . . . . . . . . . . . . . . . . . . . . . . . 19

4.2 Continuity equation and analytically weak formulation . . . . . . . . . . . . 23

References . . . . . . . . . . . . . . . . . . . . . . . . . . . 25

This article is part of the section "Theory of PDEs" edited by Eduardo Teixeira.

$凶 \quad$ P. K. Friz

friz@math.tu-berlin.de

C. Bellingeri

bellinge@math.tu-berlin.de

A. Djurdjevac

adjurdjevac@zedat.fu-berlin.de

N. Tapia

tapia@math.tu-berlin.de

1 TU Berlin, Berlin, Germany

2 FU Berlin, Berlin, Germany

3 WIAS, Berlin, Germany 


\section{Introduction}

We consider the transport equation, here posed (w.l.o.g.) as terminal value problem. This is,

$$
\begin{cases}-\partial_{t} u(t, x)=\sum_{i=1}^{d} f_{i}(x) \cdot D_{x} u(t, x) \dot{W}_{t}^{i} \equiv \Gamma u_{t}(x) \dot{W}_{t} & \text { in } \quad(0, T) \times \mathbb{R}^{n}, \\ u=g & \text { on } \quad\{T\} \times \mathbb{R}^{n} .\end{cases}
$$

for fixed $T>0$, with vector fields $f=\left(f_{1}, \ldots, f_{d}\right)$ driven by a $\mathrm{C}^{1}$-driving signal $W=$ $\left(W^{1}, \ldots, W^{d}\right)$. The canonical pairing of $D u=D_{x} u=\left(\partial_{x^{1}} u, \ldots, \partial_{x^{n}} u\right)$ with a vector field is indicated by a dot, and we already used the operator / vector notation

$$
\Gamma_{i}=f_{i}(x) \cdot D_{x}, \quad \Gamma=\left(\Gamma_{1}, \ldots, \Gamma_{d}\right) .
$$

By the methods of characteristics, the unique (classical) $\mathcal{C}^{1,1}$ transport solution $u:[0, T] \times$ $\mathbb{R}^{n} \rightarrow \mathbb{R}$, is given explicitly by

$$
u(s, x)=u(s, x ; W):=g\left(X_{T}^{s, x}\right),
$$

provided $g \in \mathcal{C}^{1}$ and the vector fields $f_{1}, \ldots, f_{d}$ are nice enough $\left(\mathcal{C}_{b}^{1}\right.$ will do) to ensure a $\mathcal{C}^{1}$ solution flow for the ODE

$$
\left\{\begin{array}{l}
\dot{X}_{t}^{s, x}=\sum_{i=1}^{d} f_{i}\left(X_{t}^{s, x}\right) \dot{W}_{t}^{i} \equiv f\left(X_{t}\right) \dot{W}_{t}, \\
X_{s}^{s, x}=x
\end{array}\right.
$$

In turn, solving this ODE with random initial data induces a natural evolution of measures, given by the continuity - or forward equation

$$
\begin{cases}\partial_{t} \rho=\sum_{i=1}^{d} \operatorname{div}_{x}\left(f_{i}(x) \rho_{t}\right) \mathrm{d} W_{t}^{i} & \text { in } \quad(0, T) \times \mathbb{R}^{n}, \\ \rho(0)=\mu & \text { on } \quad\{0\} \times \mathbb{R}^{n} .\end{cases}
$$

Well-posedness of the "trinity" transport/flow/continuity will depend on the regularity of the data. For $W \in \mathcal{C}^{1}$ we have an effective vector field

$$
b(t, x)=\sum_{i=1}^{d} f_{i}(x) \dot{W}_{t}^{i}
$$

which is continuous in $t \in[0, T]$ and inherits the regularity of $f$. In particular, $f \in \mathrm{C}^{1}$ will be sufficient for a $e^{1,1}$-flow. In a landmark paper, DiPerna-Lions [9] and then Ambrsosio [1], showed that the transport problem (weak solutions) is well-posed under bounds on $\operatorname{div} b$ (rather than $D_{x} b$ ) which in turn leads to a generalized flow. Another fundamental direction may be called regularisation by noise, based on the observation that generically $\dot{X}=$ $f_{0}(X)+($ noise $)$ is much better behaved than the noise-free problem, see e.g. [2,4,6,10,12,21].

Our work is not concerning with DiPerna-Lions type analysis, nor regularisation by noise. In fact, our driving vector fields will be very smooth, to compensate for the the irregularity of the noise, which we here assumed to be very rough (This trade-off is typical in rough paths and regularity structures.)

Specifically, we continue a programme started independently by Bailleul-Gubinelli [3] (see also [8]) and Diehl et al. [7] and take $W$ as a rough path, henceforth called W. As 
in these works, we are interested in an intrinsic notion of solution (Rough path stability of transport problems was already noted in [5]). The contribution of this article is a treatment of rough noise of arbitrarily low regularity. Based on a suitable definition of solution, carefully introduced below, we can show

Theorem 1.1 Assume $\mathbf{W}$ is a weakly geometric rough path of Hölder regularity with exponent $\gamma \in(0,1]$. Assume $f$ has $2\left\lfloor\gamma^{-1}\right\rfloor+1$ bounded derivatives. Then there is a unique spatially regular (resp. measure-valued) solution to the rough transport (resp. continuity) equation with regular terminal data (resp. measure-valued initial data).

This should be compared with [3,7], which both treat the "level-2 case", with Hölder noise of exponent $\gamma>1 / 3$. Treating the general case, i.e. with arbitrarily small Hölder exponent, requires us in particular to fully quantify the interaction of iterated integrals, themselves constrained by shuffle-relations, and the controlled structure of the PDE problem at hand. In fact, the shuffle relations will be seen crucial to preserve the hyperbolic nature of the rough transport equation. This is different for (ordinary) rough differential equations where the shuffle relations can be discarded at the price of working with branched (think: Itô-type) rough paths. For what it's worth, our arguments restricted to the (well-known) level-2-case still contain some worthwhile simplifications with regard to the existing literature, e.g. by avoiding the analysis of an adjoint equation [7] and showing uniqueness for weak solutions of the continuity equations via a small class of test functions. On our way we also (have to) prove some facts on (controlled) geometric rough paths of independent interest, not (or only in the branched setting [16,18]) available in the literature.

Relation to existing works: Unlike the case of rough transport equation, when it comes to stochastic constructions it is impossible to mention all related works stretching over more than four decades, from e.g. Funaki [13], Ogawa [23] to recent works such as [24] with fractional noise and Russo-Valois integration.

The many benefits of a robust theory of stochastic partial differential equations, by combining a deterministic RPDE theory with Brownian and more general noise, are now well documented and need not be repeated in detail. Let us still recall one example of interest: multidimensional fractional Brownian motion admits a canonical geometric rough path lift (see e.g. [11]) $1 / 4<\alpha<\mathrm{H}$, which constitutes an admissible rough noise for our rough transport and continuity equations. Various authors (see for example Unterberger [26], Nualart and Tindel [22], etc.) have constructed "renormalised" canonical fractional Brownian rough paths for any $H>0$, fully covered by Theorem 1.1 .

\section{Notations}

We fix once and for all a time $T>0$. In what follows we abbreviate estimates of the form $|(a)-(b)| \lesssim|t-s|^{\gamma}$ by writing $(a) \underset{\gamma}{=}(b)$. Given $\gamma \in(0,1)$ we denote by $\mathcal{C}^{\gamma}$ the classical Hölder space, i.e. consisting of functions $f:[0, T] \rightarrow \mathbb{R}$ such that

$$
\sup _{t \neq s} \frac{\left|f_{t}-f_{s}\right|}{|t-s|^{\gamma}}<\infty \text {. }
$$

Throughout the paper we say geometric rough path, when we really mean weakly geometric rough path (since we only work with this type of rough path, the difference [14] will not matter to us). 


\section{Rough paths}

We start by reviewing the definition of geometric rough paths of roughness $\gamma \in(0,1)$ and controlled rough paths. We will do so in a Hopf-algebraic language following [18], but before we will introduce some basic concepts.

A word of length $p \geq 1$ over the alphabet $\{1, \ldots, d\}$ is a tuple $w=\left(i_{1}, \ldots, i_{p}\right) \in$ $\{1, \ldots, d\}^{p}$, and we set $|w|:=p$. We denote by $\varepsilon$ the empty word, which is by convention the unique word with zero length. Given two non-empty words $v=\left(i_{1}, \ldots, i_{p}\right)$ and $w=$ $\left(i_{p+1}, \ldots, i_{p+q}\right)$, we denote by $v w:=\left(i_{1}, \ldots, i_{p}, i_{p+1}, \ldots, i_{p+q}\right)$ their concatenation. By definition $\varepsilon w=w \varepsilon=w$. We observe that in any case $|v w|=|v|+|w|$. The concatenation product is associative but not commutative.

The symmetric group $\mathbb{S}_{p}$ acts on words of length $p$ by permutation of its entries, that is, $\sigma . w:=\left(i_{\sigma(1)}, \ldots, i_{\sigma(p)}\right)$. Given two integers $p, q \geq 1$, a $(p, q)$-shuffle is a permutation $\sigma \in \mathbb{S}_{p+q}$ such that

$$
\sigma(1)<\sigma(2)<\cdots<\sigma(p) \text { and } \sigma(p+1)<\sigma(p+2)<\cdots<\sigma(p+q) .
$$

We denote by $\operatorname{Sh}(p, q)$ the set of all $(p, q)$-shuffles.

\subsection{The shuffle algebra}

The shuffle product was introduced by Ree [25] to study the combinatorial properties of iterated integrals, following K.-T. Chen's work. Let $d \geq 1$ be fixed, and consider the tensor algebra $H$ over $\mathbb{R}^{d}$, which is defined to be the direct sum

$$
H:=\bigoplus_{p=0}^{\infty}\left(\mathbb{R}^{d}\right)^{\otimes p}
$$

A linear basis for $H$ is given by pure tensors $e_{i_{1}} \otimes \cdots \otimes e_{i_{p}}, p \geq 1$ where $\left\{e_{1}, \ldots, e_{d}\right\}$ is a basis of $\mathbb{R}^{d}$, and the additional element 1 which generates $\left(\mathbb{R}^{d}\right)^{\otimes 0}:=\mathbb{R} \mathbf{1}$. In order to ease the notation we denote, for a word $w=\left(i_{1}, \ldots, i_{p}\right), e_{w}:=e_{i_{1}} \otimes e_{i_{2}} \otimes \cdots \otimes e_{i_{p}}$. By definition, the set $\left\{e_{w}:|w|=p\right\}$ is a linear basis for $\left(\mathbb{R}^{d}\right)^{\otimes p}$ for any $p \geq 0$.

The space $H$ is endowed with a product $\amalg: H \otimes H \rightarrow H$, called the shuffle product, defined on pure tensors as

$$
e_{i_{1} \cdots i_{p}} \uplus e_{i_{p+1} \cdots i_{p+q}}=\sum_{\sigma \in \operatorname{Sh}(p, q)} e_{\sigma .\left(i_{1}, \ldots, i_{p+q}\right)} .
$$

There is also another operation, called the deconcatenation coproduct $\Delta: H \rightarrow H \otimes H$, defined by

$$
\Delta e_{w}:=\sum_{u v=w} e_{u} \otimes e_{v}
$$

The shuffle product and the deconcatenation coproduct satisfy a compatibility relation (which will not play any role in the sequel), turning the tripe $(H, \omega, \Delta)$ into a graded connected bialgebra. This implies the existence of a linear map $S: H \rightarrow H$, called the antipode, turning $(H, \amalg, \Delta, S)$ into a Hopf algebra. In our particular setting, $S$ can be explicitly computed on basis elements by $S\left(e_{i_{1} \cdots i_{p}}\right)=(-1)^{p} e_{i_{p} \cdots i_{1}}$. 
The coproduct endows the dual space $H^{*}$ with an algebra structure via the convolution product given, for $g, h \in H^{*}$, by

$$
\langle g \star h, x\rangle:=\langle g \otimes h, \Delta x\rangle .
$$

On pure tensor this yields

$$
\left\langle g \star h, e_{w}\right\rangle=\sum_{u v=w}\left\langle g, e_{u}\right\rangle\left\langle h, e_{v}\right\rangle .
$$

A character is a linear map $g \in H^{*}$ such that $\langle g, x ш y\rangle=\langle g, x\rangle\langle g, y\rangle$ for all $x, y \in H$. It is a standard result (see e.g. [20]) that the collection of all characters on $H$ forms a group $G$ under the convolution product whose identity is the function $\mathbf{1}^{*} \in H^{*}$, defined by $\mathbf{1}^{*}\left(e_{b}\right)=0$ for every word $b$ and $\mathbf{1}^{*}(\mathbf{1})=1$. The inverse of an element $g \in G$ can be computed by using the antipode: $g^{-1}=g \circ S$.

Given $N \geq 0$, we consider the step- $N$ truncated tensor algebra

$$
H_{N}=\bigoplus_{p=0}^{N}\left(\mathbb{R}^{d}\right)^{\otimes p} .
$$

Definition 2.1 A step- $N$ truncated character is a linear map $g \in H_{N}^{*}$ such that

$$
\langle g, x \amalg y\rangle=\langle g, x\rangle\langle g, y\rangle
$$

for all $x \in\left(\mathbb{R}^{d}\right)^{\otimes p}$ and $y \in\left(\mathbb{R}^{d}\right)^{\otimes q}$ with $p+q \leq N$.

It is not hard to show that the set $G^{(N)}$ of all step- $N$ truncated characters is also a group under the convolution product, whose identity is again $\mathbf{1}^{*}$. Denoting by $e_{1}^{*}, \ldots, e_{d}^{*}$ the basis of $\mathbb{R}^{d}$ dual to $\left\{e_{1}, \ldots, e_{d}\right\}$, we introduce the dual basis $\left(e_{a}^{*}\right)$ of $H_{N}^{*}$ in the canonical way, that is, for a word $w$ we denote by $e_{w}^{*}$ the unique linear map on $H_{N}$ such that

$$
\left\langle e_{w}^{*}, e_{v}\right\rangle=\delta_{w}(v) .
$$

The convolution product of two of these basis elements can be explicitly computed. Indeed, by definition

$$
\left\langle e_{u}^{*} \star e_{v}^{*}, e_{w}\right\rangle=\sum_{u^{\prime} v^{\prime}=w}\left\langle e_{u}^{*}, e_{u^{\prime}}\right\rangle\left\langle e_{v}^{*}, e_{v^{\prime}}\right\rangle
$$

which is nonzero if and only if $w=u v$, in which case $\left\langle e_{u}^{*} \star e_{v}^{*}, e_{w}\right\rangle=1$. Therefore $e_{u}^{*} \star e_{v}^{*}=$ $e_{u v}^{*}$. For this reason this product is also known as the concatenation product.

\subsection{Geometric rough paths}

We now recall the notion of geometric rough paths. The group $G^{(N)}$ can be endowed with a sub-additive homogeneous norm $\|\cdot\|_{N}: G^{(N)} \rightarrow \mathbb{R}_{+}$, see [19] for further details. This allows us to define a left invariant metric on $G^{(N)}$ by setting

$$
d_{N}(g, h):=\left\|h^{-1} g\right\|_{N} .
$$

Definition 2.2 Let $N_{\gamma}:=\left\lfloor\gamma^{-1}\right\rfloor$ denote the integer part of $\gamma^{-1}$. A geometric rough path of regularity $\gamma$ is a $\gamma$-Hölder path $\mathbf{W}:[0, T] \rightarrow\left(G^{\left(N_{\gamma}\right)}, d_{N}\right)$. The set of all geometric rough paths of regularity $\gamma$ will be denoted by $\mathscr{C}^{\gamma}$. 
By definition of the increments $\mathbf{W}_{s t}:=\mathbf{W}_{s}^{-1} \star \mathbf{W}_{t}$ satisfy the so-called Chen's relations

$$
\mathbf{W}_{s t}=\mathbf{W}_{s u} \star \mathbf{W}_{u t}
$$

for all $0 \leq s, u, t \leq T$. Moreover, by construction of the homogeneous norm $\|\cdot\|_{N}$, for any word $w$ such that $|w| \leq N_{\gamma}$ one has

$$
\sup _{t \neq s} \frac{\left|\left\langle\mathbf{W}_{s t}, e_{w}\right\rangle\right|}{|t-s|^{|w| \gamma}}<\infty .
$$

\subsection{Controlled rough paths and rough integrals}

One of the main goals of rough paths theory is to give meaning to solutions of controlled equations of the form

$$
\mathrm{d} X_{t}=\sum_{i=1}^{d} f_{i}\left(X_{t}\right) \mathrm{d} \mathbf{W}_{t}^{i},
$$

for some collection of sufficiently regular vector fields $f_{1}, \ldots, f_{d}$ on $\mathbb{R}^{n}$ and where the driving signals $W^{1}, \ldots, W^{d}$ are very irregular. The general philosophy is that if the smoothness of the vector fields compensates the lack of regularity of the driving signals, then we can still have existence of solutions given that we reinterpret the equation in the appropriate sense. The central ingredient for proving this kind of results is the notion of controlled rough path which we now recall.

Definition $2.3[11,16]$ Let $\mathbf{W} \in \mathscr{C}^{\gamma}$ and $1 \leq N \leq N_{\gamma}+1$. A rough path controlled by $\mathbf{W}$ is a path $\mathbf{X}:[0, T] \rightarrow H_{N-1}$ if for any word $w$ such that $|w| \leq N-1$ the path $t \mapsto\left\langle e_{w}^{*}, \mathbf{X}_{t}\right\rangle \in \mathrm{C}^{\gamma}$ and

$$
\left\langle e_{w}^{*}, \mathbf{X}_{t}\right\rangle_{(N-|w|) \gamma}^{=}\left\langle\mathbf{W}_{s t} \star e_{w}^{*}, \mathbf{X}_{s}\right\rangle
$$

for all $s<t$. We denote by $\mathscr{D}_{\mathbf{W}}^{N \gamma}$ the (vector) space of paths $\mathbf{X}$ satisfying (2.6).

We say that a path $X:[0, T] \rightarrow \mathbb{R}$ is controlled by $\mathbf{W}$ if there exists a controlled path $\mathbf{X} \in \mathscr{D}_{\mathbf{W}}^{N \gamma}$ such that $\left\langle\mathbf{1}^{*}, \mathbf{X}_{t}\right\rangle=X_{t}$; we call $\mathbf{X}$ a controlled rough path above (the controlled path) $X$.

Remark 2.4 The definition in [11] seems more restrictive in that one always take $N=N_{\gamma}$, which is the minimal value of $N$ required for rough integration. The case $N=N_{\gamma}+1$ is convenient to keep track of the additional information obtained by rough integration, see Remark 2.7.

Remark 2.5 Alternatively, by writing $\mathbf{X}$ and $\mathbf{W}$ as the sums

$$
\mathbf{X}_{s}=\sum_{|u| \leq N-1}\left\langle e_{u}^{*}, \mathbf{X}_{s}\right\rangle e_{u}, \quad \mathbf{W}_{s t}=\sum_{|v| \leq N}\left\langle\mathbf{W}_{s t}, e_{v}\right\rangle e_{v}^{*},
$$

the condition in Eq. (2.6) can be explicitly written

$$
\left\langle e_{w}^{*}, \mathbf{X}_{t}\right\rangle \underset{(N-|w|) \gamma}{=} \sum_{0 \leq|v| \leq N-|w|}\left\langle e_{v w}^{*}, \mathbf{X}_{s}\right\rangle\left\langle\mathbf{W}_{s t}, e_{v}\right\rangle,
$$

for any word $w$. 
By construction of the vector space $\mathscr{D}_{\mathbf{W}}^{N \gamma}$, the quantity

$$
\|\mathbf{X}\| \mathbf{W} ; N \gamma:=\sum_{0 \leq|w|<N} \sup _{s<t} \frac{\left|\left\langle e_{w}^{*}, \mathbf{X}_{t}\right\rangle-\left\langle\mathbf{W}_{s t} \star e_{w}^{*}, \mathbf{X}_{s}\right\rangle\right|}{|t-s|^{(N-|w|) \gamma}},
$$

is finite for any $\mathbf{X} \in \mathscr{D}_{\mathbf{W}}^{N \gamma}$. We can easily show that $\|\cdot\|_{\mathbf{W} ; N \gamma}$ is a seminorm, and $\mathscr{D}_{\mathbf{W}}^{N \gamma}$ becomes a Banach space under the norm

$$
\|\mathbf{X}\|_{\mathscr{D}_{\mathbf{W}}^{N \gamma}}:=\max _{|w| \leq N-1}\left|\left\langle e_{w}^{*}, \mathbf{X}_{0}\right\rangle\right|+\|\mathbf{X}\|_{\mathbf{W} ; N \gamma} .
$$

We extend the notion of controlled rough path above a vector-valued path $X:[0, T] \rightarrow \mathbb{R}^{n}$. In this case, the path $\mathbf{X}$ takes values in $\left(H_{N-1}\right)^{n}$, that is, each component path $\left\langle e_{w}^{*}, \mathbf{X}\right\rangle$ is a vector of $\mathbb{R}^{n}$, which we denote by

$$
\left\langle e_{w}^{*}, \mathbf{X}\right\rangle=\left(\left\langle e_{w}^{*}, \mathbf{X}\right\rangle_{1}, \ldots,\left\langle e_{w}^{*}, \mathbf{X}\right\rangle_{n}\right) .
$$

Then we require the bound in Eq. (2.6) to hold componentwise, or equivalently, we can replace the absolute value of the left-hand side by any norm on $\mathbb{R}^{n}$. We denote this space by $\left(\mathscr{D}_{\mathbf{W}}^{N \gamma}\right)^{n}$.

Using the higher-order information contained in the controlled rough path $\mathbf{X} \in \mathscr{D}_{\mathbf{W}}^{N \gamma}$, we recall the rigorous notion of rough integral of $\mathbf{X}$ against $\mathbf{W}$. For its proof see [11].

Theorem 2.6 Let $\mathbf{W} \in \mathscr{C}^{\gamma}$ and $\mathbf{X} \in \mathscr{D}_{\mathbf{W}}^{N_{\gamma} \gamma}$. For every $i \in\{1, \ldots, d\}$ there exists a unique real valued path in $\mathrm{e}^{\gamma}$

$$
t \mapsto \int_{0}^{t} X_{u} \mathrm{~d} \mathbf{W}_{u}^{i}:=\lim _{|\pi| \rightarrow 0} \sum_{[a, b] \in \pi} \sum_{0 \leq|w| \leq N_{\gamma}-1}\left\langle e_{w}^{*}, \mathbf{X}_{a}\right\rangle\left\langle\mathbf{W}_{a b}, e_{w i}\right\rangle,
$$

where $\pi$ is a sequence of partitions of $[0, t]$ whose mesh $|\pi|$ converges to 0 . We call it the rough integral of $X$ with respect to $W^{i}$. Moreover one has the estimate

$$
\int_{0}^{t} X_{u} \mathrm{~d} \mathbf{W}_{u}^{i}-\int_{0}^{s} X_{u} \mathrm{~d} \mathbf{W}_{u}^{i}=: \int_{s}^{t} X_{u} \mathrm{~d} \mathbf{W}_{u}^{i} \underset{\left(N_{\gamma}+1\right) \gamma}{=} \sum_{0<|w| \leq N_{\gamma}-1}\left\langle e_{w}^{*}, \mathbf{X}_{s}\right\rangle\left\langle\mathbf{W}_{s t}, e_{w i}\right\rangle
$$

for any $s<t$. Introducing the function $\int_{0} \mathbf{X}_{u} \mathrm{~d} \mathbf{W}_{u}^{i}:[0, T] \rightarrow H_{N_{\gamma}}$ given by

$$
\left\langle\mathbf{1}^{*}, \int_{0}^{t} \mathbf{X}_{u} \mathrm{~d} \mathbf{W}_{u}^{i}\right\rangle:=\int_{0}^{t} X_{u} \mathrm{~d} \mathbf{W}_{u}^{i},\left\langle e_{w i}^{*}, \int_{0}^{t} \mathbf{X}_{u} \mathrm{~d} \mathbf{W}_{u}^{i}\right\rangle:=\left\langle e_{w}^{*}, \mathbf{X}_{t}\right\rangle
$$

and zero elsewhere, one has $\int_{0}^{.} \mathbf{X}_{u} \mathrm{~d} \mathbf{W}_{u}^{i} \in \mathscr{D}_{\mathbf{W}}^{\left(N_{\gamma}+1\right) \gamma}$.

Remark 2.7 Differently from the general definition of the $\mathscr{D}_{\mathbf{W}}^{N \gamma}$ spaces, in order to define the rough integral it is necessary to start from a controlled rough path $\mathbf{X} \in \mathscr{D}_{\mathbf{W}}^{N_{\gamma} \gamma}$. The operation of integration on controlled rough path comes also with some quantitative bounds. Looking at the definition, it is also possible to prove there exists a constant $C(T, \gamma, \mathbf{W})>0$ depending on $T, \gamma, \mathbf{W}$ such that

$$
\left\|\int_{0}^{\cdot} \mathbf{X}_{u} \mathrm{~d} \mathbf{W}_{u}^{i}\right\|_{\mathscr{D}_{\mathbf{W}}^{\left(N_{\gamma}+1\right) \gamma}} \leq C(T, \gamma, \mathbf{W})\|\mathbf{X}\|_{\mathscr{D}_{\mathbf{W}}^{N \gamma}}^{N_{\gamma}}
$$

Therefore the application $\mathbf{X} \mapsto \int \mathbf{X} \mathrm{d} \mathbf{W}^{i}$ is a continuous linear map. 
The second operation we introduce is the composition of a controlled rough path and a smooth function. Given a smooth function $\phi: \mathbb{R}^{n} \rightarrow \mathbb{R}$, its $k$-th derivative at $x \in \mathbb{R}^{n}$ is the multilinear map $D^{k} \phi(x):\left(\mathbb{R}^{n}\right)^{\otimes k} \rightarrow \mathbb{R}$ such that for $v^{1}, \ldots, v^{k} \in \mathbb{R}^{n}$,

$$
D^{k} \phi(x)\left(v^{1}, \ldots, v^{k}\right)=\sum_{\alpha_{1}, \ldots, \alpha_{k}=1}^{n} \frac{\partial^{k} \phi}{\partial x_{\alpha_{1}} \cdots \partial x_{\alpha_{k}}}(x) v_{\alpha_{1}}^{1} \cdots v_{\alpha_{k}}^{k} .
$$

To ease notation we define

$$
\partial^{\alpha} \phi(x):=\frac{\partial^{k} \phi}{\partial x_{\alpha_{1}} \cdots \partial x_{\alpha_{k}}}(x)=\frac{\partial^{k} \phi}{\partial x_{1}^{i_{1}} \cdots \partial x_{n}^{i_{n}}}(x)
$$

for a word $\alpha=\left(\alpha_{1}, \ldots, \alpha_{k}\right) \in\{1, \ldots, n\}^{k}$; of course, such $\alpha$ induces a multi-index $i=$ $\left(i_{1}, \ldots, i_{n}\right) \in \mathbb{N}^{n}$, where $i_{j}$ counts the number of entries of $\alpha$ that equal $j$.

We note that $D^{k} \phi(x)$ is symmetric, meaning that for any permutation $\sigma \in \mathbb{S}_{k}$ we have that $D^{k} \phi(x)\left(v^{1}, \ldots, v^{k}\right)=D^{k} \phi(x)\left(v^{\sigma(1)}, \ldots, v^{\sigma(k)}\right)$.

Remark 2.8 Observe that we also use the notion of word in this case, albeit with a different alphabet. In order to avoid confusion we reserve latin letters such as $u, v, w$, etc for words on the alphabet $\{1, \ldots, d\}$, introduced in the beginning of Sect. 2, and greek letters such as $\alpha, \beta$, etc for words on the alphabet $\{1, \ldots, n\}$ as above.

With these notations, Taylor's theorem states that if $\phi: \mathbb{R}^{n} \rightarrow \mathbb{R}^{m}$ is of class $\mathrm{C}^{r+1}\left(\mathbb{R}^{n}, \mathbb{R}^{m}\right)$ then for any $j=1, \ldots, m$ one has the identity

$$
\phi^{j}(y)=\sum_{k=0}^{r} \frac{1}{k !} D^{k} \phi^{j}(x)\left((y-x)^{\otimes k}\right)+\mathrm{O}\left(|y-x|^{r+1}\right) .
$$

In what follows, for any finite number of words $u_{1}, \ldots, u_{k}$ we introduce the set

$$
\operatorname{Sh}\left(u_{1}, \ldots, u_{k}\right):=\left\{w:\left\langle e_{w}^{*}, e_{u_{1}} ш \ldots ш e_{u_{k}}\right\rangle \neq 0\right\} .
$$

Since the shuffle product is commutative, for any permutation $\sigma \in \mathbb{S}_{k}$ we have that

$$
\operatorname{Sh}\left(u_{1}, \ldots, u_{k}\right)=\operatorname{Sh}\left(u_{\sigma(1)}, \ldots, u_{\sigma(k)}\right) .
$$

Thanks to this notation, we can prove Faà di Bruno's formula (see also [17]). We denote by $\mathcal{P}(m)$ the collection of all partitions of $\{1, \ldots, m\}$. Given $\pi=\left\{B_{1}, \ldots, B_{p}\right\} \in \mathcal{P}(m)$, we let $\# \pi:=p$ denote the number of its blocks, and for each block we denote by $|B|$ its cardinality.

Lemma 2.9 For any couple of functions $g: \mathbb{R}^{n} \rightarrow \mathbb{R}^{n}$ and $f: \mathbb{R}^{n} \rightarrow \mathbb{R}$ sufficiently smooth and every $m \geq 1$, letting $h:=f \circ g$ one has the identity

$$
D^{m} h(x)\left(v_{1}, \ldots, v_{m}\right)=\sum_{\pi \in \mathcal{P}(m)} D^{\# \pi} f(g(x))\left(D^{\left|B_{1}\right|} g(x)\left(v_{B_{1}}\right), \ldots, D^{\left|B_{p}\right|} g(x)\left(v_{B_{p}}\right)\right)
$$

where $v_{B}:=\left(v_{i_{1}}, \ldots, v_{i_{q}}\right)$ for $B=\left\{i_{1}, \ldots, i_{q}\right\}$.

In particular, for any word $\alpha=\left(\alpha_{1}, \ldots, \alpha_{m}\right)$ we have

$$
\partial^{\alpha} h(x)=\sum_{k=1}^{m} \frac{1}{k !} \sum_{\substack{\beta_{1}, \ldots, \beta_{k} \\ \alpha \in \operatorname{Sh}\left(\beta_{1}, \ldots, \beta_{k}\right)}} D^{k} f(g(x))\left(\partial^{\beta_{1}} g(x), \ldots, \partial^{\beta_{k}} g(x)\right) .
$$


Proof We proceed by induction on $m$. For $m=1$ the formula reads

$$
D h(x) v=D f(g(x)) D g(x) v
$$

which is the usual chain rule. Suppose the formula holds for some $m \geq 1$. Then, applying the chain rule to each of the terms we get

$$
\begin{aligned}
D^{m+1} h(x)\left(v_{1}, \ldots, v_{m+1}\right) \\
=\sum_{\pi \in \mathcal{P}(m)} \sum_{l=1}^{k} D^{\# \pi+1} f(g(x))\left(D^{\left|B_{1}\right|} g(x) v_{B_{1}}, \ldots, D^{\left|B_{l}\right|+1} g(x)\left(v_{B_{l}}, v_{m+1}\right), \ldots, D^{\left|B_{k}\right|} g(x) v_{B_{k}}\right) \\
\quad+\sum_{\pi \in \mathcal{P}(m)} D^{\# \pi+1} f(g(x))\left(D^{\left|B_{1}\right|} g(x) v_{B_{1}}, \ldots, D^{\left|B_{k}\right|} g(x) v_{B_{k}}, D g(x) v_{m+1}\right) \\
=\sum_{\pi^{\prime} \in \mathcal{P}(m+1)} D^{\# \pi^{\prime}} f(g(x))\left(D^{\left|B_{1}^{\prime}\right|} g(x)\left(v_{B_{1}^{\prime}}\right), \ldots, D^{\left|B_{p}^{\prime}\right|} g(x)\left(v_{B_{k^{\prime}}^{\prime}}\right)\right)
\end{aligned}
$$

where the last identity follows from the fact that every partition $\pi^{\prime} \in \mathcal{P}(m+1)$ can be obtained by either appending $m+1$ to one of the blocks of some partition $\pi \in \mathcal{P}(m)$ or by adding the singleton block $\{m+1\}$ to it.

Given a word $\alpha=\left(\alpha_{1}, \ldots, \alpha_{m}\right)$, we evaluate the previous formula in the canonical basis vectors $v_{1}=e_{\alpha_{1}}, \ldots, v_{m}=e_{\alpha_{m}}$ to obtain

$$
\begin{aligned}
\partial^{\alpha} h(x) & =D^{m} h(x)\left(v_{1}, \ldots, v_{m}\right) \\
& =\sum_{\pi \in \mathcal{P}(m)} D^{\# \pi} f(g(x))\left(\partial^{\alpha_{B_{1}}} g(x), \ldots, \partial^{\alpha_{B_{k}}} g(x)\right)
\end{aligned}
$$

where $\alpha_{B}=\left(\alpha_{i_{1}}, \ldots, \alpha_{i_{q}}\right)$ if $B=\left\{i_{1}, \ldots, i_{p}\right\}$. It is now clear that for any choice of $\pi \in \mathcal{P}(m)$ the words $\alpha_{B_{1}}, \ldots, \alpha_{B_{k}}$ satisfy $\alpha \in \operatorname{Sh}\left(\alpha_{B_{1}}, \ldots, \alpha_{B_{k}}\right)$. Conversely, if $\alpha \in \operatorname{Sh}\left(\beta_{1}, \ldots, \beta_{k}\right)$, there is a partition $\pi=\left\{B_{1}, \ldots, B_{k}\right\}$ with $B_{j}=$ is such that $\beta_{j}=\alpha_{B_{j}}$. Moreover, for any choice of such a partition, any of the $k$ ! permutations of its blocks result in the same evaluation by symmetry of the differential. Thus

$$
\partial^{\alpha} h(x)=\sum_{k=1}^{m} \frac{1}{k !} \sum_{\substack{\beta_{1}, \ldots, \beta_{k} \\ \alpha \in \operatorname{Sh}\left(\beta_{1}, \ldots, \beta_{k}\right)}} D^{k} f(g(x))\left(\partial^{\beta_{1}} g(x), \ldots, \partial^{\beta_{k}} g(x)\right) .
$$

Remark 2.10 This result should be well-known to experts, yet the closest reference we found in the literature [17] only covers the scalar case (and does not immediately yield the multivariate case).

Using a similar technique we show a version of this identity for controlled rough paths.

Theorem 2.11 Let $\mathbf{W} \in \mathscr{C}^{\gamma}, 1 \leq N \leq N_{\gamma}+1, \mathbf{X} \in\left(\mathscr{D}_{\mathbf{W}}^{N \gamma}\right)^{n}$, and $\phi \in C^{N}\left(\mathbb{R}^{n}, \mathbb{R}^{m}\right)$ and set $X_{t}:=\left\langle\mathbf{1}, \mathbf{X}_{t}\right\rangle$. We introduce the path $\boldsymbol{\Phi}(\mathbf{X}):[0, T] \rightarrow\left(H_{N-1}\right)^{m}$ defined by $\left\langle\mathbf{1}^{*}, \boldsymbol{\Phi}(\mathbf{X})_{t}\right\rangle_{j}=$ $\phi^{j}\left(X_{t}\right)$ and for any $j=1, \ldots, m$, and any non-empty word $w$ by the identity

$$
\left\langle e_{w}^{*}, \boldsymbol{\Phi}(\mathbf{X})_{t}\right\rangle_{j}:=\sum_{k=1}^{|w|} \frac{1}{k !} \sum_{\substack{w_{1}, \ldots, w_{k} \\ w \in \operatorname{Sh}\left(w_{1}, \ldots, w_{k}\right)}} D^{k} \phi^{j}\left(X_{t}\right)\left(\left\langle e_{w_{1}}^{*}, \mathbf{X}_{t}\right\rangle, \ldots,\left\langle e_{w_{k}}^{*}, \mathbf{X}_{t}\right\rangle\right) .
$$

Then $\boldsymbol{\Phi}(\mathbf{X})$ is also a controlled rough path belonging to $\left(\mathscr{D}_{\mathbf{W}}^{N \gamma}\right)^{m}$. 
Remark 2.12 A similar statement in the setting of branched rough paths [16, Lemma 8.4] is known and somewhat easier due to the absence of shuffle relations.

Before going into the proof, we introduce some more notation. If $\mathbf{X}$ is a controlled path, $L \in \mathcal{L}\left(\left(\mathbb{R}^{n}\right)^{\otimes k}, \mathbb{R}^{m}\right), t \geq 0$ and $w_{1}, \ldots, w_{k}$ are words, we let

$$
L\left(t ; w_{1}, \ldots, w_{k}\right):=L\left(\left\langle e_{w_{1}}^{*}, \mathbf{X}_{t}\right\rangle, \ldots,\left\langle e_{w_{k}}^{*}, \mathbf{X}_{t}\right\rangle\right) .
$$

Proof It is sufficient to prove the result when $m=1$. We first prove the result for the case of $\left\langle\mathbf{1}^{*}, \boldsymbol{\Phi}(\mathbf{X})_{t}\right\rangle=\phi\left(X_{t}\right)$. By Taylor expanding $\phi$ up to order $N$ around $X_{s}$ we get that

$$
\phi\left(X_{t}\right) \underset{N \gamma}{=} \sum_{k=0}^{N-1} \frac{1}{k !} D^{k} \phi\left(X_{s}\right)\left(\left(X_{t}-X_{s}\right)^{\otimes k}\right) .
$$

Since $\mathbf{X} \in\left(\mathscr{D}_{\mathbf{W}}^{N \gamma}\right)^{n}$, according to Remark 2.5, we have

$$
\left\langle\mathbf{1}^{*}, \mathbf{X}_{t}-\mathbf{X}_{s}\right\rangle \underset{N \gamma}{=}\left\langle\mathbf{W}_{s t}-\mathbf{1}^{*}, \mathbf{X}_{s}\right\rangle=\sum_{0<|w|<N}\left\langle e_{w}^{*}, \mathbf{X}_{s}\right\rangle\left\langle\mathbf{W}_{s t}, e_{u}\right\rangle .
$$

Plugging this estimate into the above equation and using the character property of $\mathbf{W}_{s t}$ in (2.2) we obtain

$$
\begin{aligned}
\phi\left(X_{t}\right) & \underset{N \gamma}{=} \sum_{k=0}^{N-1} \frac{1}{k !} \sum_{u_{1}, \ldots, u_{k}} D^{k} \phi\left(X_{s}\right)\left(s ; w_{1}, \ldots, w_{k}\right)\left\langle\mathbf{W}_{s t}, e_{w_{1}} ш \cdots ш e_{w_{k}}\right\rangle \\
& =\sum_{k=0}^{N-1} \frac{1}{k !} \sum_{w_{1}, \ldots, w_{k}} \sum_{|w| \leq N} D^{k} \phi\left(X_{s}\right)\left(s ; w_{1}, \ldots, w_{k}\right)\left\langle e_{w}^{*}, e_{w_{1}} \uplus \cdots \uplus e_{w_{k}}\right\rangle\left\langle\mathbf{W}_{s t}, e_{w}\right\rangle
\end{aligned}
$$

so the desired estimate follows.

Now we show the bound (2.6) for all words $w \neq 1$. By fixing an integer $1 \leq k \leq|w|$ and words $u_{1}, \ldots, u_{k}$ such that $w \in \operatorname{Sh}\left(u_{1}, \ldots, u_{k}\right)$ we consider the term

$$
D^{k} \phi\left(X_{t}\right)\left(t ; w_{1}, \ldots, w_{k}\right) .
$$

Again, since $\mathbf{X}$ is controlled by $\mathbf{W}$, plugging the estimate in Remark 2.5 into (2.16) and using the multilinearity of the derivative we obtain

$$
D^{k} \phi\left(X_{t}\right)\left(t ; w_{1}, \ldots, w_{k}\right) \underset{(N-|w|) \gamma}{=} \sum_{v_{1}, \ldots, v_{k}} D^{k} \phi\left(X_{t}\right)\left(s ; v_{1} w_{1}, \ldots, v_{k} w_{k}\right)\left\langle\mathbf{W}_{s t}, e_{v_{1}} \uplus \cdots \uplus e_{v_{k}}\right\rangle .
$$

Performing a Taylor expansion of $D^{k} \phi$ up to order $N-|w|$ between $X_{t}$ and $X_{s}$, we obtain

$$
\begin{aligned}
& D^{k} \phi\left(X_{t}\right)\left(s ; v_{1} w_{1}, \ldots, v_{k} w_{k}\right) \\
& \underset{(N-|w|) \gamma}{=} \sum_{m=0}^{N-|w|-1} \frac{1}{m !} D^{k+m} \phi\left(X_{s}\right)\left(\left(X_{t}-X_{s}\right)^{\otimes m},\left\langle e_{v_{1} w_{1}}^{*}, \mathbf{X}_{s}\right\rangle, \ldots,\left\langle e_{v_{k} w_{k}}^{*}, \mathbf{X}_{s}\right\rangle\right) .
\end{aligned}
$$


Combining the estimates (2.17) and (2.18) with (2.15) into the definition of $\left\langle e_{w}^{*}, \boldsymbol{\Phi}(\mathbf{X})_{t}\right\rangle$, we obtain the identity

$$
\begin{aligned}
& \left\langle e_{w}^{*}, \boldsymbol{\Phi}(\mathbf{X})_{t}\right\rangle \\
& \underset{(N-|w|) \gamma}{=} \sum_{k=1}^{|w|} \sum_{m=0}^{N-1-|w|} \sum_{\substack{w_{1}, \ldots, w_{k} \\
w \in \operatorname{Sh}\left(u_{1}, \ldots, u_{k}\right)}} \sum_{\substack{v_{1}, \ldots, v_{k} \\
z_{1}, \ldots, z_{m}}} \frac{1}{k ! m !} D^{k+m} \phi\left(X_{S}\right)\left(v_{1} w_{1}, \ldots, v_{k} w_{k}, z_{1}, \ldots, z_{m}\right) \\
& \quad \times\left\langle\mathbf{W}_{s t}, e_{v_{1}} \uplus \cdots \uplus e_{v_{k}} \amalg e_{z_{1}} \uplus \cdots \uplus e_{z_{m}}\right\rangle .
\end{aligned}
$$

Since the derivative $D^{k+m} \phi\left(X_{S}\right)$ is symmetric we can replace it with

$$
\frac{k ! m !}{(k+m) !} \sum_{I_{k} \sqcup J_{m}=\{1, \ldots, m+k\}} D^{k+m} \phi\left(X_{s}\right)\left(v_{i_{1}} w_{i_{1}}, \ldots, z_{j_{1}}, \ldots, v_{i_{k}} w_{i_{k}}, \ldots\right) .
$$

Replacing this expression in the right-hand side of (2.19), it is now an easy but tedious exercise to verify the resulting expression is equal to the sum

$$
\sum_{0 \leq|u|<N-|w|} \sum_{l=1}^{|w|+|u|} \sum_{\substack{u_{1}, \ldots, u_{l} \\ u w \in \operatorname{Sh}\left(u_{1}, \ldots, u_{l}\right)}} \frac{1}{l !} D^{l} \phi\left(X_{s}\right)\left(s ; u_{1}, \ldots, u_{l}\right)\left\langle\mathbf{W}_{s t}, e_{u}\right\rangle .
$$

Thereby proving the result.

Remark 2.13 A similar proof gives quantitative bounds on the application $\mathbf{X} \rightarrow \boldsymbol{\Phi}(\mathbf{X})$. Indeed for any $\phi \in \mathcal{C}_{b}^{N}\left(\mathbb{R}^{n}, \mathbb{R}^{m}\right)$ it is possible to prove that this application is locally Lipschitz on $\mathscr{D}_{\mathbf{W}}^{N \gamma}$.

\section{Rough differential equations}

Now we come to the definition of solution of the RDE

$$
\left\{\begin{array}{l}
\mathrm{d} X_{t}=\sum_{i=1}^{d} f_{i}\left(X_{t}\right) \mathrm{d} \mathbf{W}_{t}^{i}, \\
X_{0}=x
\end{array}\right.
$$

We assume that the vector fields $f_{1}, \ldots, f_{d}$ are of class at least $\mathrm{C}^{N_{\gamma}}$, so that by Theorem 2.11 the composition $f_{i}\left(X_{t}\right)$ can be lifted to a controlled path $\mathbf{F}_{i}:\left(\mathscr{D}_{\mathbf{W}}^{N_{\gamma} \gamma}\right)^{n} \rightarrow\left(\mathscr{D}_{\mathbf{W}}^{N_{\gamma} \gamma}\right)^{n}$.

Definition 3.1 A path $X:[0, T] \rightarrow \mathbb{R}^{n}$ is a solution of (3.1) if there exists a controlled path $\mathbf{X} \in\left(\mathscr{D}_{\mathbf{W}}^{N_{\gamma} \gamma}\right)^{n}$ satisfying $\left\langle\mathbf{1}^{*}, \mathbf{X}_{t}\right\rangle=X_{t}$ such that

$$
\mathbf{X}_{t}-\mathbf{X}_{s}=\sum_{i=1}^{d} \int_{s}^{t} \mathbf{F}_{i}(\mathbf{X})_{u} \mathrm{~d} \mathbf{W}_{u}^{i}
$$

for all $s, t \in[0, T]$.

Remark 3.2 We stress that (3.2) is an equation in $\mathscr{D}_{\mathbf{W}}^{N_{\gamma} \gamma}$, which in fact implies that $\left\langle e_{w}^{*}, \mathbf{X}_{t}\right\rangle=$ $F_{w}\left(X_{t}\right)$ for all words $w$ with $|w| \leq N_{\gamma}-1$. 
Remark 3.3 If $\mathbf{X} \in \mathscr{D}_{\mathbf{W}}^{N_{\gamma} \gamma}$ satisfies Eq. (3.2), it can also be regarded as an element of $\mathscr{D}_{\mathbf{W}}^{\left(N_{\gamma}+1\right) \gamma}$, by Eq. (2.8). Therefore we freely treat solutions to RDEs as elements of either of these spaces.

By solving a fixed point equation on $\left(\mathscr{D}_{\mathbf{W}}^{N_{\gamma} \gamma}\right)^{n}$ (see e.g. [11]) of the form

$$
\mathbf{X}_{t}=\mathbf{X}_{0}+\sum_{i=1}^{d} \int_{0}^{t} \mathbf{F}_{i}(\mathbf{X})_{u} \mathrm{~d} \mathbf{W}_{u}^{i}
$$

with (see Proposition 3.4 for the definition of the functions $F_{w}: \mathbb{R}^{n} \rightarrow \mathbb{R}^{n}$ )

$$
\mathbf{X}_{0}=\sum_{|w| \leq N_{\gamma}-1} F_{w}(x) e_{w} \in\left(H_{N_{\gamma}-1}\right)^{n},
$$

one can prove that there exists a unique global solution of (3.2) if the vector fields are of class $\mathcal{C}_{b}^{N_{\gamma}+1}$. We recall this interesting expansion of the solution.

Proposition 3.4 (Davie's expansion) A path $X:[0, T] \rightarrow \mathbb{R}^{n}$ is the unique rough path solution to Eq. (2.5) in the sense of Definition 3.1 if and only if

$$
X_{t} \underset{\left(N_{\gamma}+1\right) \gamma}{=} \sum_{0 \leq|w| \leq N_{\gamma}} F_{w}\left(X_{s}\right)\left\langle\mathbf{W}_{s t}, e_{w}\right\rangle
$$

and the coefficients of its lift $\mathbf{X} \in\left(\mathscr{D}_{\mathbf{W}}^{N_{\gamma}+1}\right)^{n}$ are given by $\left\langle e_{w}^{*}, \mathbf{X}_{t}\right\rangle=F_{w}\left(X_{t}\right)$ where the functions $F_{w}: \mathbb{R}^{n} \rightarrow \mathbb{R}^{n}$ are recursively defined by by $F_{\varepsilon}:=\mathrm{id}$ and

$$
F_{i w}(x):=D F_{w}(x) f_{i}(x) .
$$

Remark 3.5 By Eq. (2.7) this results actually implies the chain of estimates, for all words $|w| \leq N_{\gamma}$,

$$
F_{w}\left(X_{t}\right) \underset{\left(N_{\gamma}+1-|w|\right) \gamma}{=} \sum_{0 \leq|u| \leq N-|w|} F_{u w}\left(X_{S}\right)\left\langle\mathbf{W}_{s t}, e_{u}\right\rangle .
$$

Proof of Proposition 3.4 Suppose that $\mathbf{X}$ is a rough solution to Eq. (2.5) in the sense of Definition 3.1. We define the functions $F_{w}: \mathbb{R}^{n} \rightarrow \mathbb{R}^{n}$ recursively by $F_{i}(x):=f_{i}(x)$ and

$$
F_{w i}(x):=\sum_{k=1}^{|w|} \frac{1}{k !} \sum_{\substack{u_{1}, \ldots, u_{k} \\ w \in \operatorname{Sh}\left(u_{1}, \ldots, u_{k}\right)}} D^{k} f_{i}(x)\left(F_{u_{1}}(x), \ldots, F_{u_{k}}(x)\right) .
$$

Now it is an easy but tedious verification to show that these functions satisfy $F_{i w}(x)=$ $D F_{w}(x) f_{i}(x)$; this identity essentially amounts to a reiterated use of the Leibniz rule. The form of the coefficients of $\mathbf{X}$ is shown by induction, it being clear for a single letter $i=$ $1, \ldots, d$. If $w$ is any word with $0 \leq|w| \leq N-1$ and $i \in\{1, \ldots, d\}$ by definition

$$
\begin{aligned}
\left\langle e_{w i}^{*}, \mathbf{X}_{t}-\mathbf{X}_{0}\right\rangle & =\left\langle e_{w i}^{*}, \sum_{j=1}^{d} \int_{0}^{t} \mathbf{F}_{j}(\mathbf{X})_{u} \mathrm{~d} W_{u}^{j}\right\rangle \\
& =\left\langle e_{w}^{*}, \mathbf{F}_{i}(\mathbf{X})_{t}\right\rangle
\end{aligned}
$$


where, in the second identity we have used Eq. (2.10). By Theorem 2.11, the last coefficient equals

$$
\sum_{k=1}^{|w|} \frac{1}{k !} \sum_{\substack{u_{1}, \ldots, u_{k} \\ w \in \operatorname{Sh}\left(u_{1}, \ldots, u_{k}\right)}} D^{k} f_{i}\left(X_{t}\right)\left(t ; u_{1}, \ldots, u_{k}\right)=F_{w i}\left(X_{t}\right)
$$

by the induction hypothesis. Then we obtain Eq. (3.3) from Definition 2.3 and Remark 2.5.

Conversely, suppose that $X$ admits the local expansion in Eq. (3.3) and that the path $\mathbf{X}$ satisfies $\left\langle e_{w}^{*}, \mathbf{X}_{t}\right\rangle=F_{w}\left(X_{t}\right)$ for all words $w$ with $|w| \leq N$. First we show that $X$ is controlled by $\mathbf{W}$ with coefficients given by $\mathbf{X}$. For this we have to Taylor expand the difference $F_{w}\left(X_{t}\right)-F_{w}\left(X_{S}\right)$ and collect terms as in the proof of Theorem 2.11. Then, by Eq. (2.10) it is not difficult to see that in fact

$$
\left\langle e_{w i}^{*}, \mathbf{X}_{t}\right\rangle=F_{w i}\left(X_{t}\right)=\left\langle e_{w i}^{*}, \sum_{j=1}^{d} \int_{0}^{t} \mathbf{F}_{j}(\mathbf{X})_{u} \mathrm{~d} W_{u}^{j}\right\rangle
$$

so that Definition 3.1 is satisfied.

\subsection{Differentiability of the flow}

It is a standard result in classical ODE theory that given a regular enough vector field $V$, the equation $\dot{X}=V(X)$ induces a smooth flow on $\mathbb{R}^{d}$. Indeed, if we let $X_{t}^{x}$ denote the unique solution of this equation such that $X_{0}^{x}=x$, then the map $(t, x) \mapsto X_{t}^{x}$ is a flow, in the sense that $\left(t, X_{s}^{x}\right) \mapsto X_{t+s}^{x}$ and the mapping $x \mapsto X_{t}^{x}$ is a diffeomorphism for each fixed $t$. More precesily, if $V$ is of class $\mathrm{C}^{k}$, then the application $x \mapsto X_{t}^{x}$ is also of class $\mathrm{C}^{k}$.

Now we show that a similar statement is true in the case of RDEs. The statement is the following

Theorem 3.6 Let $f_{1}, \ldots, f_{d}$ be a family of class $\mathrm{C}_{b}^{N_{\gamma}+1+k}$ vector fields in $\mathbb{R}^{d}$ for some integer $k \geq 0$, and $\mathbf{W} \in \mathscr{C}^{\gamma}$. Then

\section{1. the RDE}

$$
\mathrm{d} X_{t}=\sum_{i=1}^{d} f_{i}\left(X_{t}\right) \mathrm{d} \mathbf{W}_{t}^{i}, \quad X_{s}=x
$$

has a unique solution $\mathbf{X}^{s, x} \in \mathscr{D}_{\mathbf{W}}^{\left(N_{\gamma}+1\right) \gamma}$,

2. the induced flow $x \mapsto X_{t}^{s, x}$ is a class $\mathrm{e}^{k+1}$ diffeomorphism for each fixed $s<t$, and

3. the partial derivatives satisfy the system of RDEs

$$
\mathrm{d} \partial^{\alpha} X_{t}^{s, x}=\sum_{i=1}^{d} \sum_{k=1}^{|\alpha|} \frac{1}{k !} \sum_{\alpha \in \operatorname{Sh}\left(\beta_{1}, \ldots, \beta_{k}\right)} D^{k} f_{i}\left(X_{t}^{s, x}\right)\left(\partial^{\beta_{1}} X_{t}^{s, x}, \ldots, \partial^{\beta_{k}} X_{t}^{s, x}\right) \mathrm{d} \mathbf{W}_{t}^{i}
$$

with initial conditions $X_{s}^{s, x}=x, \partial^{i} X_{s}^{s, x}=e_{i}$ and $\partial^{\alpha} X_{s}^{s, x}=0$ for all words with $|\alpha| \geq 2$.

Proof Points (1) and (2) are standard results in rough paths as found e.g. in Chapter 11 in [15]. For the algebraic identity in (3), it suffices to show the results in the case $W$ is smooth. Indeed, by standard arguments $\mathbf{W} \in \mathscr{C}^{\gamma}$ can be approximated uniformly with uniform $\gamma$ Hölder rough path bound, and hence in $\mathscr{C}^{\gamma-\eta}$ for any $\eta>0$, while on the other hand the 
particular structure (cf. Chapter 11 in [15]) of the system of (rough) differential equations guarantees uniqueness and global existence so that the limiting argument is justified.

It remains to show point (3) for $\mathbf{W}$ smooth. We note that the integral representation of the solution

$$
X_{t}^{s, x}=x+\sum_{i=1}^{d} \int_{s}^{t} f_{i}\left(X_{u}^{s, x}\right) \dot{W}_{u}^{i} \mathrm{~d} u
$$

holds. By Lemma 2.9, for any $\alpha=\left(\alpha_{1}, \ldots, \alpha_{m}\right)$ and $s<u<t$, we have

$$
\partial^{\alpha} X_{u t}^{s, x}=\int_{u}^{t} \sum_{i=1}^{d} \sum_{k=1}^{m} \frac{1}{k !} \sum_{\beta_{1}, \ldots, \beta_{k}} D^{k} f_{i}\left(X_{r}^{s, x}\right)\left(\partial^{\beta_{1}} X_{r}^{s, x}, \ldots, \partial^{\beta_{k}} X_{r}^{s, x}\right) \dot{W}_{r}^{i} \mathrm{~d} r
$$

which is the smooth version of Eq. (3.6).

We aim now to obtain a Davie-type expansion of the partial derivatives $\partial^{\alpha} X^{s, x}$ by making use of point 3. above. We observe that the above system of equations has the form

$$
\begin{aligned}
\mathrm{d} X_{t}^{s, x} & =\sum_{i=1}^{d} f_{i}\left(X_{t}^{s, x}\right) \mathrm{d} \mathbf{W}_{t}^{i} \\
\mathrm{~d} D X_{t}^{s, x} & =\sum_{i=1}^{d} D f_{i}\left(X_{t}^{s, x}\right) D X_{t}^{s, x} \mathrm{~d} \mathbf{W}_{t}^{i} \\
\mathrm{~d} D^{2} X_{t}^{s, x} & =\sum_{i=1}^{d} D f_{i}\left(X_{t}^{s, x}\right) D^{2} X_{t}^{s, x} \mathrm{~d} \mathbf{W}_{t}^{i}+(\ldots)
\end{aligned}
$$

with initial conditions $X_{s}^{s, x}=x, D X_{s}^{s, x}=I, D^{2} X_{s}^{s, x}=D^{3} X_{s}^{s, x}=\cdots=0$, where the inhomogeneity (...) is not important to spell out.

The expansion is clear only for the first equation; it is just Eq. (3.3). We would like to use Proposition 3.4 to obtain an expansion of the second equation but the problem is that the vector field driving the equation depends on time, so the result does not directly apply. For the third and subsequent equations the problem is not only that but also they are non-homogeneous.

To solve this problem we extend our state space $\mathbb{R}^{n}$ to (the still finite-dimensional space)

$$
\mathfrak{S}_{k}:=\mathbb{R}^{n} \oplus \mathcal{L}\left(\mathbb{R}^{n}, \mathbb{R}^{n}\right) \oplus \cdots \oplus \mathcal{L}\left(\left(\mathbb{R}^{n}\right)^{\otimes(k-1)}, \mathbb{R}^{n}\right)
$$

and define the vector fields (we give a more precise definition below in Eq. (3.8)) $\mathfrak{f}_{i}: \mathfrak{S}_{k} \rightarrow$ $\mathfrak{S}_{k}$ by

$$
\mathfrak{f}_{i}(\mathfrak{x}):=\left(f_{i}(x), D f_{i}(x)\left(y_{1}\right), D^{2} f_{i}(x)\left(y_{1}, y_{1}\right)+D f_{i}(x)\left(y_{2}\right), \ldots\right)
$$

where $\mathfrak{x}=\left(x, y_{1}, y_{2}, \ldots, y_{k-1}\right) \in \mathfrak{S}_{k}$. The previous proposition shows that if

$$
\mathfrak{X}_{t}^{s, x}:=\left(X_{t}^{s, x}, D X_{t}^{s, x}, \ldots, D^{k-1} X_{t}^{s, x}\right)
$$

then

$$
\mathrm{d} \mathfrak{X}_{t}^{s, x}=\sum_{i=1}^{d} \mathfrak{f}_{i}\left(\mathfrak{X}_{t}^{s, x}\right) \mathrm{d} \mathbf{W}_{t}^{i}, \quad \mathfrak{X}_{s}^{s, x}:=\mathfrak{x}=(x, I, 0, \ldots, 0) .
$$


This transformation turns the system of non-autonomous non-homogeneous RDEs into a single autonomous homogeneous $\mathrm{RDE}$ in $\mathfrak{S}_{k}$.

Corollary 3.7 For any word $\alpha$, the partial derivatives of the solution flow $X^{s, x}$ have the following Davie expansion: for any $p=1, \ldots, k-1$,

$$
D^{p} X_{t}^{s, x} \underset{\left(N_{\gamma}+1\right) \gamma}{=} \sum_{0 \leq|v| \leq N_{\gamma}} D^{p} F_{w}(x)\left\langle\mathbf{W}_{s t}, e_{w}\right\rangle .
$$

In particular, for a word $\alpha \in\{1, \ldots, n\}^{p}$ we have that

$$
\partial^{\alpha} X_{t}^{s, x} \underset{\left(N_{\gamma}+1\right) \gamma}{=} \sum_{0 \leq|v| \leq N_{\gamma}} \partial^{\alpha} F_{w}(x)\left\langle\mathbf{W}_{s t}, e_{w}\right\rangle .
$$

Proof The hypotheses on the vector fields $f_{1}, \ldots, f_{d}$ imply that $\mathfrak{f}_{1}, \ldots, \mathfrak{f}_{d}$ are of class $\mathrm{C}_{b}^{N_{\gamma}+1}$ on $\mathfrak{S}_{k}$, so this equation has a unique solution. Applying Proposition 3.4 in this extended space we obtain, for $s<t$, the expansion

$$
\mathfrak{X}_{t}^{s, x} \underset{\left(N_{\gamma}+1\right) \gamma}{=} \sum_{0 \leq|w|<N} \mathfrak{F}_{w}(\mathfrak{x})\left\langle\mathbf{W}_{s t}, e_{w}\right\rangle .
$$

In order to deduce the result, we need to show that $\mathfrak{F}_{w}(\mathfrak{x})_{p}=D^{p} F_{w}(x)$ for all words $w$ and $p=0,1, \ldots, k-1$. We do this by induction on the length of $w$. If $w=i$ is a single letter, the $p$-th component, $p=0,1, \ldots, k-1$, of the vector field $\mathfrak{f}_{i}$ is given by $\mathfrak{f}_{i}(x)_{0}=f_{i}(x)$ and

$$
\mathfrak{f}_{i}(\mathfrak{x})_{p}=\sum_{j=1}^{p} \sum_{(r)_{j}} \frac{p !}{r_{1} ! \cdots r_{j} !(1 !)^{r_{1}} \cdots(j !)^{r_{k}}} D^{p-j+1} f_{i}(x)\left(y_{1}^{r_{1}}, \ldots, y_{j}^{r_{j}}\right)
$$

where the inner sum is over the set of indices $\left(r_{1}, \ldots, r_{j}\right)$ such that $r_{1}+\cdots+r_{j}=p-j+1$ and $r_{1}+2 r_{2}+\cdots+j r_{j}=p$. For our particular initial condition, $y_{j}=0$ for $j=2,3, \ldots, k-1$ the formula simplifies to

$$
\mathfrak{f}_{i}(\mathfrak{x})_{p}=D^{p} f_{i}(x) \in \mathcal{L}\left(\left(\mathbb{R}^{n}\right)^{\otimes p}, \mathbb{R}^{n}\right)
$$

since the only term left in (3.8) is the one with $j=1, r_{1}=p$.

We continue by induction on the length of the word. We compute the $p$-th derivative of $x \mapsto F_{i w}(x)=D F_{w}(x) f_{i}(x)$ by recognizing that $F_{i w}=\varphi_{1} \circ \varphi_{2}$ with $\varphi_{1}(x, h)=D F_{w}(x) h$ and $\varphi_{2}(x)=\left(x, f_{i}(x)\right)$. A quick check gives that the higher order derivatives of $\varphi_{1}$ and $\varphi_{2}$ are given by

$$
\begin{aligned}
D^{m} \varphi_{1}(x, h)\left(\left(u_{1}, v_{1}\right), \ldots,\left(u_{m}, v_{m}\right)\right)= & D^{m+1} F_{w}(x)\left(u_{1}, \ldots, u_{m}, h\right) \\
& +\sum_{j=1}^{m} D^{m} F_{w}(x)\left(u_{1}, \ldots, \hat{u}_{j}, \ldots, u_{m}\right) \\
D^{m} \varphi_{2}(x)\left(h_{1}, \ldots, h_{m}\right)= & \left(h_{1} \delta_{m=1}, D^{m} f_{i}(x)\left(h_{1}, \ldots, h_{m}\right)\right)
\end{aligned}
$$

where $\hat{u}_{j}=v_{j}$. Thus, using Lemma 2.9 we get that

$$
D^{p} F_{i w}(x)\left(h_{1}, \ldots, h_{p}\right)=\sum_{\pi \in \mathcal{P}(p)} D^{\# \pi} \varphi_{1}\left(\varphi_{2}(x)\right)\left(D^{\left|B_{1}\right|} \varphi_{2}(x) h_{B_{1}}, \ldots, D^{\left|B_{q}\right|} \varphi_{2}(x) h_{B_{q}}\right) .
$$

Now we have three cases, depending on the number of blocks of the partition in the above summation: 
1. $q=p$ : there is a single partition with $p$ blocks, and each block is a singleton. In this case the term equals

$$
D^{p+1} F_{w}(x)\left(h_{1}, \ldots, h_{p}, f_{i}(x)\right)+\sum_{j=1}^{p} D^{p} F_{w}(x)\left(h_{1}, \ldots, D f_{i}(x) h_{j}, \ldots, h_{p}\right) .
$$

2. $q=1$ : there is a single partition with one block, namely $\pi=\{1, \ldots, p\}$. In this case the term equals

$$
D F_{w}(x)\left[D^{p} f_{j}(x)\left(h_{1}, \ldots, h_{p}\right)\right] .
$$

3. $1<q<p$ : there is at least one block of size greater than one, which means that the first term in the expression for $D^{m} \varphi_{1}$ vanishes since at least one of $u_{1}, \ldots, u_{m}$ vanishes. For the rest of the terms, the exact result depends on whether there is a block with exactly one block or not: if all blocks have more than one block then the whole expression vanishes; otherwise, we obtain one term for each of the blocks having size exactly one, and it is of the form

$$
D^{\# \pi} F_{w}(x)\left(h_{B}, D^{\# p-|B|} f_{i}(x) h_{\pi \backslash B}\right) .
$$

In either case, using the induction hypothesis it is possible to show that each of the terms appearing are of the form $\partial_{r} \mathfrak{F}_{w}(\mathfrak{x})_{p} \mathfrak{f}_{i}(\mathfrak{x})_{r}$, which then means that $D^{p} F_{i w}(x)=$ $\left[D \mathfrak{F}_{w}(\mathfrak{x}) \mathfrak{f}_{i}(\mathfrak{x})\right]_{p}$ as desired. For example, the term

$$
D^{p+1} F_{w}\left(h_{1}, \ldots, h_{p}, f_{i}(x)\right)
$$

corresponds to

$$
\left[\partial_{0} \mathfrak{F}_{w}(\mathfrak{x})_{p} \mathfrak{f}_{i}(\mathfrak{x})_{0}\right]\left(h_{1}, \ldots, h_{p}\right)
$$

and so on.

In particular for the first derivative, the first few terms of the expansion read

$$
\begin{aligned}
D X_{t}^{s, x}= & I+\sum_{i=1}^{d} D f_{i}(x)\left\langle\mathbf{W}_{s t}, e_{i}\right\rangle \\
& +\sum_{i, j=1}^{d}\left(D f_{j}(x) D f_{i}(x)+D^{2} f_{j}(x)\left(f_{i}(x), \text { id }\right)\right)\left\langle\mathbf{W}_{s t}, e_{i j}\right\rangle+\cdots
\end{aligned}
$$

\subsection{Itô's formula for RDEs}

The last ingredient to add in the study of the rough transport equation is to write down a change of variable formula for a solution of Eq. (2.5) for some sufficiently smooth vector field $f=\left(f_{1}, \ldots, f_{d}\right)$. By analogy with terminology of stochastic calculus we call it an "Itô formula". For any $i=1, \ldots, n$ we denote by $\Gamma_{i}$ the differential operator $f_{i}(x) \cdot D_{x}$ and for any non-empty word $w=i_{1} \ldots i_{m}$ we use the shorthand notation

$$
\Gamma_{w}:=\Gamma_{i_{1}} \circ \cdots \circ \Gamma_{i_{m}} .
$$

Moreover we adopt the convention $\Gamma_{\varepsilon}=\mathrm{id}$. 
Lemma 3.8 Let $f_{1}, \ldots, f_{d} \in \mathcal{e}^{N_{\gamma}+1}\left(\mathbb{R}^{n} ; \mathbb{R}^{n}\right)$ be vector fields on $\mathbb{R}^{n}$. If $\phi: \mathbb{R}^{n} \rightarrow \mathbb{R}$ is a smooth function and $w$ is a nonempty word, then

$$
\Gamma_{w} \phi(x)=\sum_{k=1}^{|w|} \frac{1}{k !} \sum_{\substack{u_{1}, \ldots, u_{k} \\ w \in \operatorname{Sh}\left(u_{1}, \ldots, u_{k}\right)}} D^{k} \phi(x)\left(F_{u_{1}}(x), \ldots, F_{u_{k}}(x)\right) .
$$

Proof Before commencing we introduce some notation. If $\phi: \mathbb{R}^{n} \rightarrow \mathbb{R}$ and $g_{1}, \ldots, g_{k}: \mathbb{R}^{n} \rightarrow$ $\mathbb{R}^{n}$ are smooth functions, we define

$$
D^{k} \phi(x):\left(g_{1}, \ldots, g_{k}\right):=D^{k} \phi(x)\left(g_{1}(x), \ldots, g_{k}(x)\right)
$$

where the right-hand side was defined in Eq. (2.11). The Leibniz rule then gives that for any $h \in \mathbb{R}^{n}$ we have

$$
\begin{aligned}
h \cdot D_{x}\left(D^{k} \phi(x):\left(g_{1}, \ldots, g_{k}\right)\right)= & D^{k+1} \phi(x):\left(h, g_{1}, \ldots, g_{k}\right) \\
& +\sum_{i=1}^{k} D^{k} \phi(x):\left(g_{1}, \ldots,\left(D_{x} g_{i}\right) h, \ldots, g_{k}\right) .
\end{aligned}
$$

We now prove the result by induction on the word's length $|w|$. If $w=i$ is a single letter then $\Gamma_{i} \phi(x)=f_{i}(x) \cdot \nabla \phi(x)=D \phi(x) f_{i}(x)$ which is exactly Eq. (3.9). Supposing the identity true for any word $w^{\prime}$ such that $\left|w^{\prime}\right| \leq|w|$, we prove it for $j w$ where $j \in\{1, \ldots, d\}$. By induction one has

$$
\Gamma_{w} \phi(x)=\sum_{k=1}^{|w|} \frac{1}{k !} \sum_{w \in \operatorname{Sh}\left(u_{1}, \ldots, u_{k}\right)}^{u_{1}, \ldots, u_{k}} D^{k} \phi(x)\left(F_{u_{1}}(x), \ldots, F_{u_{k}}(x)\right) .
$$

By the above form of Leibniz rule, with $g_{i}=F_{u_{i}}$ and $h=f_{j}(x)$, and noticing that by definition

$$
D_{x} F_{u_{i}}(x) f_{j}(x)=F_{j u_{i}}(x)
$$

we obtain that

$$
\begin{aligned}
\Gamma_{j}\left(D^{k} \phi(x):\left(F_{u_{1}}, \ldots, F_{u_{k}}\right)\right)= & D^{k+1} \phi(x):\left(f_{j}, F_{u_{1}}, \ldots, F_{u_{k}}\right) \\
& +\sum_{i=1}^{k} D^{k} \phi(x):\left(F_{u_{1}}, \ldots, F_{j u_{i}}, \ldots, F_{u_{k}}\right) .
\end{aligned}
$$

Summing this expression over words $u_{1}, \ldots, u_{k}$, we can rewrite it as

$$
\begin{aligned}
& \sum_{r=1}^{k} \sum_{\substack{u_{1}, \ldots, u_{k} \\
j w \in \operatorname{Sh}\left(u_{1}, \ldots, j u_{r}, \ldots, u_{k}\right)}} D^{k} \phi(x):\left(F_{u_{1}}, \ldots, F_{j u_{r}}, \ldots, F_{u_{k}}\right) \\
& +\frac{1}{k+1} \sum_{r=1}^{k+1} \sum_{\substack{u_{1}, \ldots, u_{k} \\
j w \in \operatorname{Sh}\left(u_{1}, \ldots, j, \ldots, u_{k}\right)}} D^{k+1} \phi(x):(F_{u_{1}}, \ldots, \overbrace{f_{j}}^{r \text { th place }}, \ldots, F_{u_{k}}),
\end{aligned}
$$


the factor $1 /(k+1)$ is introduced because of the symmetry of $D^{k+1} \phi(x)$. Summing finally over $k$, we can express the final expression as

$$
\begin{aligned}
\Gamma_{j w} \phi(x)= & \sum_{k=1}^{|w|} \frac{1}{k !} \sum_{r=1}^{k} \sum_{\substack{u_{1}, \ldots, u_{k} \\
j w \in \operatorname{Sh}\left(u_{1}, \ldots, j u_{r}, \ldots, u_{k}\right)}} D^{k} \phi(x):\left(F_{u_{1}}, \cdots, F_{j u_{r}}, \ldots, F_{u_{k}}\right) \\
& +\sum_{k=1}^{|w|} \frac{1}{(k+1) !} \sum_{r=1}^{k+1} \sum_{\substack{u_{1}, \ldots, u_{k} \\
j w \in \operatorname{Sh}\left(u_{1}, \ldots, j, \ldots, u_{k}\right)}} D^{k+1} \phi(x):(F_{u_{1}}, \ldots, \overbrace{f_{j}}^{r \text { th place }}, \ldots, F_{u_{k}}) .
\end{aligned}
$$

Since the letter $j$ may appear as a single word or concatenated at the right with some word, we finally identify the whole expression above with

$$
\sum_{k=1}^{|w|+1} \frac{1}{k !} \sum_{\substack{u_{1}, \ldots, u_{k} \\ j a \in \operatorname{Sh}\left(u_{1}, \ldots, u_{k}\right)}} D^{k} \phi(x):\left(F_{u_{1}}, \ldots, F_{u_{k}}\right) .
$$

Now we show a formula for the composition of the solution to the RDE (2.5) and a sufficiently smooth function.

Theorem 3.9 (Itô formula for RDEs) Let $f_{i} \in \mathrm{C}^{N_{\gamma}+1}$ and let $\mathbf{X} \in \mathscr{D}_{\mathbf{W}}^{\left(N_{\gamma}+1\right) \gamma}$ be the unique solution of Eq. (2.5) and $X_{t}=\left\langle\mathbf{1}^{*}, \mathbf{X}_{t}\right\rangle$. Then for any real valued function $\phi \in \mathcal{C}_{b}^{N_{\gamma}+1}\left(\mathbb{R}^{n}\right)$ one has the identity

$$
\phi\left(X_{t}\right)=\phi\left(X_{s}\right)+\sum_{i=1}^{d} \int_{s}^{t}\left(\Gamma_{i} \phi\right)\left(X_{r}\right) \mathrm{d} \mathbf{W}_{r}^{i}
$$

More generally, one has the following estimates at the level of controlled rough paths

$$
\left\langle e_{w}^{*}, \boldsymbol{\Phi}(\mathbf{X})_{t}\right\rangle \underset{\left(N_{\gamma}+1-|w|\right) \gamma}{=}\left\langle e_{w}^{*}, \boldsymbol{\Phi}(\mathbf{X})_{s}\right\rangle+\left\langle e_{w}^{*}, \sum_{i=1}^{d} \int_{s}^{t}\left(\Gamma_{i} \boldsymbol{\Phi}\right)(\mathbf{X})_{r} \mathrm{~d} \mathbf{W}_{r}^{i}\right\rangle,
$$

where $\Gamma_{i} \boldsymbol{\Phi}(\mathbf{X})$ is the controlled lift of composition of $\mathbf{X}$ with the function $\Gamma_{i} \phi \in \mathrm{e}^{N_{\gamma}}$ and any non-empty word such that $|w| \leq N_{\gamma}$.

Proof of Theorem 3.9 The theorem is obtained by comparing the coefficients of the controlled rough paths $\boldsymbol{\Phi}(\mathbf{X})_{t}$ and $\int_{0}^{t}\left(\Gamma_{i} \boldsymbol{\Phi}\right)\left(X_{r}\right) \mathrm{d} \mathbf{W}_{r}^{i}$ for every $i=1, \ldots, d$. Using Lemma 3.8 and Proposition 3.4 , for every non-empty word $a$ one has

$$
\begin{aligned}
\left\langle e_{w}^{*}, \boldsymbol{\Phi}(\mathbf{X})_{t}\right\rangle & =\sum_{k=1}^{|w|} \frac{1}{k !} \sum_{\substack{u_{1}, \ldots, u_{k} \\
w \in \operatorname{Sh}\left(u_{1}, \ldots, u_{k}\right)}} D^{k} \phi\left(X_{t}\right)\left(t ; u_{1}, \ldots, u_{k}\right) \\
& =\sum_{k=1}^{|w|} \frac{1}{k !} \sum_{\substack{u_{1}, \ldots, u_{k} \\
w \in \operatorname{Sh}\left(u_{1}, \ldots, u_{k}\right)}} D^{k} \phi\left(X_{t}\right):\left(F_{u_{1}}, \ldots, F_{u_{k}}\right) \\
& =\Gamma_{w} \phi\left(X_{t}\right) .
\end{aligned}
$$


Using the same identities we also deduce for any word $w$,

$$
\left\langle e_{w j}^{*}, \sum_{i=1}^{d} \int_{0}^{t}\left(\Gamma_{i} \boldsymbol{\Phi}\right)(\mathbf{X})_{r} \mathrm{~d} \mathbf{W}_{r}^{i}\right\rangle=\left\langle e_{w}^{*},\left(\Gamma_{j} \boldsymbol{\Phi}\right)(\mathbf{X})_{t}\right\rangle=\Gamma_{w}\left(\Gamma_{j} \phi\right)\left(X_{t}\right)=\Gamma_{w j} \phi\left(X_{t}\right) .
$$

Since $\sum_{i=1}^{n} \int_{0}^{t}\left(\Gamma_{i} \boldsymbol{\Phi}\right)(\mathbf{X})_{r} \mathrm{~d} \mathbf{W}_{r}^{i}$ and $\boldsymbol{\Phi}(\mathbf{X})_{t}$ belong both to $\mathscr{D}_{\mathbf{W}}^{\left(N_{\gamma}+1\right) \gamma}$ for any word $w$ one has both

$$
\left\langle e_{w}^{*}, \boldsymbol{\Phi}(\mathbf{X})_{t}\right\rangle-\left\langle e_{w}^{*}, \boldsymbol{\Phi}(\mathbf{X})_{s}\right\rangle \underset{\left(N_{\gamma}+1-|w|\right) \gamma}{=} \sum_{0<|v| \leq N_{\gamma}-|w|}\left\langle e_{w v}^{*}, \boldsymbol{\Phi}(\mathbf{X})_{s}\right\rangle\left\langle\mathbf{W}_{s t}, e_{v}\right\rangle,
$$

and

$\left\langle e_{w}^{*}, \sum_{i=1}^{n} \int_{s}^{t}\left(\Gamma_{i} \boldsymbol{\Phi}\right)(\mathbf{X})_{r} \mathrm{~d} \mathbf{W}_{r}^{i}\right\rangle \underset{\left(N_{\gamma}+1-|w|\right) \gamma}{=} \sum_{0<|v| \leq N_{\gamma}-|w|}\left\langle e_{w v}^{*}, \sum_{i=1}^{n} \int_{0}^{s}\left(\Gamma_{i} \boldsymbol{\Phi}\right)(\mathbf{X})_{r} \mathrm{~d} \mathbf{W}_{r}^{i}\right\rangle\left\langle\mathbf{W}_{s t}, e_{v}\right\rangle$.

The identities (3.12) and (3.13) imply that the right-hand sides of the above estimates are the same quantities. Thus we obtain Eq. (3.11) by simply subtracting one side from the other. In case $w=\mathbf{1}$ one has

$$
\phi\left(X_{t}\right)-\phi\left(X_{s}\right)-\sum_{i=1}^{d} \int_{s}^{t}\left(\Gamma_{i} \phi\right)\left(X_{r}\right) \mathrm{d} \mathbf{W}_{r}^{i} \underset{\left(N_{\gamma}+1\right) \gamma}{=} 0 .
$$

Since $\left(N_{\gamma}+1\right) \gamma>1$ and the right hand side is the increment of a path, one has the identity (3.10).

Using the identities (3.12) we can rewrite the Itô formula using only the operators $\Gamma_{w}$.

Corollary 3.10 (Itô-Davie formula for RDEs) Let $X:[0, T] \rightarrow \mathbb{R}^{n}$ be the unique solution of Eq. (2.5). Then for any real valued function $\phi \in \mathcal{C}_{b}^{N_{\gamma}+1}\left(\mathbb{R}^{n}\right)$ and any word $w$ one has the estimate

$$
\Gamma_{w} \phi\left(X_{t}\right) \underset{\left(N_{\gamma}+1-|w|\right) \gamma}{=} \sum_{0 \leq|v| \leq N_{\gamma}-|w|} \Gamma_{v w} \phi\left(X_{s}\right)\left\langle\mathbf{W}_{s t}, e_{v}\right\rangle .
$$

\section{Rough transport and continuity}

\subsection{Rough transport equation}

We now consider the rough transport equation

$$
\left\{\begin{array}{l}
-\mathrm{d} u_{s}=\sum_{i=1}^{d} \Gamma_{i} u_{s} \mathrm{~d} \mathbf{W}_{s}^{i}, \\
u(T, \cdot)=g(\cdot)
\end{array}\right.
$$

where we recall the differential operator $\Gamma_{i}:=f_{i} \cdot D_{x}$ for some vector fields $f_{1}, \ldots, f_{d}$ on $\mathbb{R}^{n}$.

We now prepare the definition of a regular solution to the rough transport equation. Since we are in the fortunate position to have an explicit solution candidate we derive a graded set of rough path estimates that provide a natural generalisation of the classical transport differential equation. 
Definition 4.1 Let $\gamma \in(0,1), \mathbf{W} \in \mathscr{C}^{\gamma}$ a weakly-geometric rough path of roughness $\gamma$ and $g \in \mathrm{C}^{N_{\gamma}+1}$. A $\mathrm{e}^{\gamma, N_{\gamma}+1}$-function $u:[0, T] \times \mathbb{R}^{n} \rightarrow \mathbb{R}$ such that $u(T, \cdot)=g(\cdot)$ is said to be a regular solution to the rough transport Eq. (4.1) if one has the estimates

$$
\Gamma_{w} u_{s}(x) \underset{\left(N_{\gamma}+1-|w|\right) \gamma}{=} \sum_{0 \leq|v| \leq N_{\gamma}-|w|} \Gamma_{w v} u_{t}(x)\left\langle\mathbf{W}_{s t}, e_{v}\right\rangle,
$$

for every $s<t \in[0, T]$, uniformly on compact sets in $x$ and any word $w$.

Remark 4.2 Since each application of the vector fields $\Gamma_{i_{1} \ldots i_{n}}$ amounts to take $n$ derivatives, these estimates have the interpretation that time regularity of $\Gamma_{i_{1} \ldots i_{n}} u$, can be traded against space regularity in a controlled sense.

Theorem 4.3 Let $f \in \mathcal{C}_{b}^{2 N_{\gamma}+1}, g \in \mathrm{C}^{N_{\gamma}+1}$ and consider the rough solution $X^{s, x}$ to Eq. (2.5). Then $u(s, x):=g\left(X_{T}^{s, x}\right)$ is a solution to the rough transport equation in the sense of Definition 4.1 .

Proof We first note that by Theorem 3.6 the map $(s, x) \mapsto X_{T}^{s, x}$ belongs to $\mathrm{C}^{\gamma, N_{\gamma}+1}$. Since $g \in \mathrm{e}^{N_{\gamma}+1}$ then $u(s, x)=g\left(X_{T}^{s, x}\right) \in \mathrm{C}^{\gamma, N_{\gamma}+1}$. Let us show that $u$ is a solution by proving the estimates given in Definition 4.1 for some fixed times $s<t<T$ and $x$ in compact set. By uniqueness of the RDE flow one has $X_{T}^{s, x}=X_{T}^{t, y}$ where $y=X_{t}^{s, x}$. Thus we deduce from the definition of $u$ the identity

$$
u_{s}(x)=u_{t}\left(X_{t}^{s, x}\right)
$$

Let $\mathbf{X}$ denote the controlled rough path such that $X_{t}^{s, x}=\left\langle\mathbf{1}^{*}, \mathbf{X}_{t}\right\rangle$. Since $g \in \mathrm{C}_{b}^{N_{\gamma}+1}$, we can apply the rough Itô formula in Eq. (3.14) to the function $x \rightarrow u_{t}(x)$ obtaining

$$
u_{t}\left(X_{t}^{s, x}\right) \underset{\left(N_{\gamma}+1\right) \gamma}{=} \sum_{|w| \leq N} \Gamma_{w} u_{t}(x)\left\langle\mathbf{W}_{s t}, w\right\rangle
$$

obtaining (4.2) for the case of $w=\varepsilon$. To show the estimates on $\Gamma_{i_{1} \ldots i_{l}} u_{s}$, we apply Lemma 3.8 to the function $x \mapsto u_{s}(x)$

$$
\Gamma_{w} u_{s}(x)=\sum_{k=1}^{|w|} \frac{1}{k !} \sum_{\substack{u_{1}, \ldots, u_{k} \\ w \in \operatorname{Sh}\left(u_{1}, \ldots, u_{k}\right)}} D^{k} u_{s}(x)\left(F_{u_{1}}(x), \ldots, F_{u_{k}}(x)\right) .
$$

Using again the identity (4.3), for any word $\alpha$ we apply Eq. (2.13) obtaining

$$
\partial^{\alpha}\left(u_{s}(x)\right)=\sum_{l=1}^{k} \frac{1}{l !} \sum_{\substack{\beta_{1}, \ldots, \beta_{l} \\ \alpha \in \operatorname{Sh}\left(\beta_{1}, \ldots, \beta_{l}\right)}} D^{l} u_{t}\left(X_{t}^{s, x}\right)\left(\partial^{\beta_{1}} X_{t}^{s, x}, \ldots, \partial^{\beta_{l}} X_{t}^{s, x}\right) .
$$

Since the vector field $f \in C_{b}^{2 N_{\gamma}+1}$ and every $\beta_{i}$ such that $\alpha \in \operatorname{Sh}\left(\beta_{1}, \ldots, \beta_{l}\right)$ satisfies $\left|\beta_{i}\right| \leq|a|$ we can apply Definition 3.7 to get

$$
\partial^{\beta_{i}} X_{t}^{s, x} \underset{\left(N_{\gamma}+1-|w|\right) \gamma}{=} \sum_{0 \leq|v| \leq N_{\gamma}-|w|} \partial^{\beta_{i}} F_{v}(x)\left\langle\mathbf{W}_{s t}, e_{v}\right\rangle .
$$


Plugging these estimates in $D^{l} u_{t}\left(X_{t}^{s, x}\right)$ and one has

$$
\begin{aligned}
& D^{l} u_{t}\left(X_{t}^{s, x}\right)\left(\partial^{\beta_{1}} X_{t}^{s, x}, \ldots, \partial^{\beta_{l}} X_{t}^{s, x}\right) \\
& \quad \sum_{\left(N_{\gamma}+1-|w|\right) \gamma} \sum_{0 \leq\left|v_{1}\right| \ldots\left|v_{l}\right| \leq N_{\gamma}-|a|} D^{l} u_{t}\left(X_{t}^{s, x}\right)\left(\partial^{\beta_{1}} F_{v_{1}}(x), \ldots, \partial^{\beta_{l}} F_{v_{l}}(x)\right)\left\langle\mathbf{W}_{s t}, e_{v_{1}} ш \cdots \varpi e_{v_{l}}\right\rangle .
\end{aligned}
$$

Plugging this expression into (4.4) we obtain

$$
\begin{aligned}
\Gamma_{w} u_{s}(x) \underset{\left(N_{\gamma}+1-|w|\right) \gamma}{=} & \sum_{k=1}^{|w|} \sum_{\substack{u_{1}, \ldots, u_{k} \\
w \in \operatorname{Sh}\left(u_{1}, \ldots, u_{k}\right)}} \sum_{\alpha_{1}, \ldots, \alpha_{k}=1}^{n} \sum_{l=1}^{k} \frac{1}{l !} \frac{1}{k !} F_{u_{1}}^{\alpha_{1}}(x) \cdots F_{u_{k}}^{\alpha_{k}}(x) \\
& \times \sum_{0 \leq\left|d_{1}\right| \ldots\left|d_{l}\right| \leq N_{\gamma}-|a|} \sum_{\substack{\beta_{1}, \ldots, \beta_{l} \\
\alpha \in \operatorname{Sh}\left(\beta_{1}, \ldots, \beta_{l}\right)}} D^{l} u_{t}\left(X_{t}^{s, x}\right)\left(\partial^{\beta_{1}} F_{v_{1}}(x), \ldots, \partial^{\beta_{l}} F_{v_{l}}(x)\right) \\
& \times\left\langle\mathbf{W}_{s t}, e_{v_{1}} \uplus \cdots \uplus e_{v_{l}}\right\rangle .
\end{aligned}
$$

Rearranging the sums and applying the definition of the functions $F_{w}$ we obtain the identity

$$
\begin{aligned}
\sum_{k=l}^{|w|} & \frac{1}{k !} \sum_{\substack{u_{1}, \ldots, u_{k} \\
w \in \operatorname{Sh}\left(u_{1}, \ldots, u_{k}\right)}} \sum_{\substack{\alpha \in \operatorname{Sh}\left(\beta_{1}, \ldots, \beta_{l}\right) \\
|\alpha|=k}} D^{l} u_{t}\left(X_{t}^{s, x}\right)\left(\partial^{\beta_{1}} F_{v_{1}}(x), \ldots, \partial^{\beta_{l}} F_{v_{l}}(x)\right) F_{u_{1}}^{\alpha_{1}}(x) \cdots F_{u_{k}}^{\alpha_{k}}(x) \\
& =\sum_{\substack{u_{1}^{\prime}, \ldots, u_{l}^{\prime} \\
w \in \operatorname{Sh}\left(u_{1}^{\prime}, \ldots, u_{l}^{\prime}\right)}} D^{l} u_{t}\left(X_{t}^{s, x}\right)\left(F_{u_{1}^{\prime} v_{1}}(x), \cdots, F_{u_{l}^{\prime} v_{l}}(x)\right) .
\end{aligned}
$$

Therefore the right-hand side of (4.6) becomes

$$
\sum_{l=1}^{|w|} \sum_{0 \leq\left|v_{1}\right| \ldots\left|v_{v}\right| \leq N_{\gamma}-|w|} \sum_{\begin{array}{c}
u_{1}^{\prime}, \ldots, u_{l}^{\prime} \\
w \in \operatorname{Sh}\left(u_{1}^{\prime}, \ldots, u_{l}^{\prime}\right)
\end{array}} \frac{1}{l !} D^{l} u_{t}\left(X_{t}^{s, x}\right)\left(F_{u_{1}^{\prime} v_{1}}(x), \cdots, F_{u_{l}^{\prime} v_{l}}(x)\right)\left\langle\mathbf{W}_{s t}, e_{v_{1}} \uplus \cdots \varpi e_{v_{l}}\right\rangle .
$$

We perform now a Taylor expansion of $D^{l} u_{t}\left(X_{t}^{s, x}\right)$ up to order $N-|w|$ between $X_{t}^{s, x}$ and $x$, yielding for any words $u_{1}^{\prime}, \ldots, u_{k}^{\prime}$

$$
\begin{aligned}
& D^{l} u_{t}\left(X_{t}^{s, x}\right)\left(F_{u_{1}^{\prime} v_{1}}(x), \cdots, F_{u_{l}^{\prime} v_{l}}(x)\right) \\
& =\underset{\left(N_{\gamma}+1-|w|\right) \gamma}{=} \sum_{m=0}^{N-|w|} \frac{1}{m !} D^{l+m} u_{t}(x)\left(\left(X_{t}^{s, x}-x\right)^{\otimes m}, F_{u_{1}^{\prime} v_{1}}(x), \cdots, F_{u_{l}^{\prime} v_{l}}(x)\right) .
\end{aligned}
$$


Plugging now the Davie expansion (3.3) truncated at order $N_{\gamma}-|w|$ into (4.7) we have the following estimate

$$
\begin{aligned}
\Gamma_{w}\left(u_{s}(x)\right) \underset{\left(N_{\gamma}+1-|w|\right) \gamma}{=} & \sum_{l=1}^{|w|} \sum_{m=0}^{N_{\gamma}-|w|} \frac{1}{l !} \frac{1}{m !} \sum_{\substack{u_{1}^{\prime}, \ldots, u_{l}^{\prime} \\
w \in \operatorname{Sh}\left(u_{1}^{\prime}, \ldots, u_{l}^{\prime}\right)}} \sum_{\substack{0 \leq\left|v_{1}\right| \ldots\left|v_{l}\right| \leq N-|w| \\
0<\left|z_{1}\right| \ldots\left|z_{m}\right| \leq N-|w|}} \\
& \times D^{l+m} u_{t}(x):\left(F_{u_{1}^{\prime} v_{1}}, \ldots, F_{z_{1}}, \ldots\right)\left\langle\mathbf{W}_{s t}, e_{v_{1}} \uplus \cdots \uplus e_{z_{1}} \uplus \ldots\right\rangle .
\end{aligned}
$$

Using the symmetry of $D^{l+m} u_{t}(x)$, we deduce

$$
\frac{l ! m !}{(l+m) !} \sum_{I_{l} \sqcup J_{m}=\{1, \ldots, m+l\}} D^{m+l} \phi(x):\left(F_{u_{i_{1}}^{\prime} v_{i_{1}}}, \cdots, F_{z_{j_{1}}}, \ldots, F_{u_{i_{l}}^{\prime} v_{i_{l}}}, \ldots\right) .
$$

Replacing this expression in the right-hand side of (4.9), we can easily verify that the resulting expression is equal to the sum

$$
\sum_{0 \leq|v| \leq N-|w|} \sum_{n=1}^{|w|+|v|} \sum_{\substack{u_{1}, \ldots, u_{n} \\ w \in \in \operatorname{Sh}\left(u_{1}, \ldots, u_{n}\right)}} \frac{1}{n !} D^{n} u_{t}(x):\left(F_{u_{1}}, \cdots, F_{u_{n}}\right)\left\langle\mathbf{W}_{s t}, e_{v}\right\rangle .
$$

Thereby proving the result.

We can now show that solutions in the sense of Definition 4.1 are unique.

Theorem 4.4 Let $f_{i} \in \mathcal{C}_{b}^{2 N_{\gamma}+1}$ with associated differential operators $\Gamma_{i}$, and $\mathbf{W} \in \mathscr{C}^{\gamma}$. Given regular terminal data $g \in \mathrm{e}^{N_{\gamma}+1}$, there exists a unique regular solution to the rough transport equation (4.1).

Proof Existence is clear, since Proposition 4.3 exactly says that $(t, x) \mapsto g\left(X_{T}^{t, x}\right)$ gives a regular solution. Let now $u$ be any solution to the rough transport equation. We show that, whenever $X=X^{\bar{s}, \bar{y}}$ for every $\bar{s}, \bar{y}$ one has the estimate

$$
u\left(t, X_{t}\right)-u\left(s, X_{s}\right) \underset{\left(N_{\gamma}+1\right) \gamma}{=} 0 .
$$

Since $\left(N_{\gamma}+1\right) \gamma>1$ this entails that $t \mapsto u\left(t, X_{t}\right)$ is constant, and so we recover the uniqueness from the identities

$$
u(s, x)=u\left(s, X_{s}^{s, x}\right)=u\left(T, X_{T}^{s, x}\right)=g\left(X_{T}^{s, x}\right) .
$$

To prove (4.10) we show that for every $k=0, \ldots, N_{\gamma}$ and any choice of indexes $i_{1}, \ldots, i_{k}$ (if $k=0$ we do not consider indexes) one has the estimates

$$
\Gamma_{i_{1} \ldots i_{k}} u_{t}\left(X_{t}\right)_{\left(N_{\gamma}+1-k\right) \gamma}^{=} \Gamma_{i_{1} \ldots i_{k}} u_{S}\left(X_{S}\right)
$$

Let us prove this estimate by reverse induction on the indices length. The case when the indices $i_{1} \ldots i_{N_{\gamma}}$ have length $N_{\gamma}$ comes easily from the algebraic manipulation

$$
\begin{aligned}
\Gamma_{i_{1} \ldots i_{N_{\gamma}}} u_{t}\left(X_{t}\right)-\Gamma_{i_{1} \ldots i_{N_{\gamma}}} u_{S}\left(X_{S}\right)= & \left(\Gamma_{i_{1} \ldots i_{N_{\gamma}}} u_{t}\left(X_{t}\right)-\Gamma_{i_{1} \ldots i_{N_{\gamma}}} u_{S}\left(X_{t}\right)\right) \\
& +\left(\Gamma_{i_{1} \ldots i_{N_{\gamma}}} u_{S}\left(X_{t}\right)-\Gamma_{i_{1} \ldots i_{N_{\gamma}}} u_{S}\left(X_{S}\right)\right) .
\end{aligned}
$$


Using the defining property of a solution in the estimates (4.2), the first difference on the righthand side is of order $\gamma$. Moreover by hypothesis on $u$ one has $\Gamma_{i_{1} \ldots i_{N \gamma}} u_{s}(\cdot) \in C^{1}$, always uniformly in $s \in[0, T]$, therefore the second difference is also of order $\gamma$, as required. Supposing the estimate true for every indices of length $k$ we will prove it on every indices $i_{1} \ldots i_{k-1}$ of length $k-1$. By repeating the same procedure as before we obtain

$$
\begin{aligned}
\Gamma_{i_{1} \ldots i_{k-1}} u_{t}\left(X_{t}\right)-\Gamma_{i_{1} \ldots i_{k-1}} u_{S}\left(X_{S}\right)= & \underbrace{\left(\Gamma_{i_{1} \ldots i_{k-1}} u_{t}\left(X_{t}\right)-\Gamma_{i_{1} \ldots i_{k-1}} u_{S}\left(X_{t}\right)\right)}_{I} \\
& +\underbrace{\left(\Gamma_{i_{1} \ldots i_{k-1}} u_{S}\left(X_{t}\right)-\Gamma_{i_{1} \ldots i_{k-1}} u_{S}\left(X_{S}\right)\right)}_{I I} .
\end{aligned}
$$

Using the definition of a solution, the first difference on the right-hand side satisfies

$$
I_{\left(N_{\gamma}+1-k\right) \gamma}^{=}-\sum_{k=1}^{N_{\gamma}+1-k} \sum_{|w|=k} \Gamma_{i_{1} \ldots i_{k-1} w} u_{t}\left(X_{t}\right)\left\langle\mathbf{W}_{s t}, w\right\rangle .
$$

On the other hand, using Lemma 3.8 two times we write $\Gamma_{i_{1} \ldots i_{k-1}} u_{s}\left(X_{t}\right)=\left\langle e_{i_{1} \cdots i_{k-1}}^{*}, \mathbf{U}_{s}(\mathbf{X})_{t}\right\rangle$ so that the second difference can be replaced by the usual remainder

$$
\begin{array}{r}
I I \underset{\left(N_{\gamma}+1-k\right) \gamma}{=} \sum_{k=1}^{N_{\gamma}+1-k} \sum_{|w|=k}\left\langle e_{i_{1} \ldots i_{k-1} w}^{*}, \mathbf{U}_{s}(\mathbf{X})_{s}\right\rangle\left\langle\mathbf{W}_{s t}, w\right\rangle \\
\begin{array}{c}
\left.N_{\gamma}+1-k\right) \gamma \\
=
\end{array} \sum_{k=1}^{N_{\gamma}+1-k} \sum_{|w|=k} \Gamma_{i_{1} \ldots i_{k-1} w} u_{s}\left(X_{s}\right)\left\langle\mathbf{W}_{s t}, w\right\rangle .
\end{array}
$$

Combining the two estimates we obtain

$$
I+I I=-\sum_{k=1}^{N_{\gamma}+1-k} \sum_{|w|=k}\left(\Gamma_{i_{1} \ldots i_{k-1} w} u_{t}\left(X_{t}\right)-\Gamma_{i_{1} \ldots i_{k-1} w} u_{S}\left(X_{S}\right)\right)\left\langle\mathbf{W}_{s t}, w\right\rangle .
$$

Since the terms in the sum involve the increment $\Gamma_{\sigma} u_{t}\left(X_{t}\right)-\Gamma_{\sigma} u_{s}\left(X_{s}\right)$ where $\sigma$ has length bigger or equal than $k$ we apply the recursive hypothesis obtaining that each term satisfies

$$
\Gamma_{i_{1} \ldots i_{k-1} w} u_{t}\left(X_{t}\right)-\Gamma_{i_{1} \ldots i_{k-1} w} u_{S}\left(X_{S}\right) \underset{\left(N_{\gamma}+1-k-|w|\right) \gamma}{=} 0
$$

and the multiplication with $\left\langle\mathbf{W}_{s t}, w\right\rangle$ gives the desired estimate.

\subsection{Continuity equation and analytically weak formulation}

Given a finite measure $\rho \in \mathcal{M}\left(\mathbb{R}^{n}\right)$ and a continuous bounded function $\phi \in C_{b}\left(\mathbb{R}^{n}\right)$, we write $\rho(\phi)=\int \phi(x) \rho(d x)$ for the natural pairing. We are interested in measure-valued (forward) solutions to the continuity equation

$$
\begin{cases}\mathrm{d}_{t} \rho_{t}=\sum_{i=1}^{d} \operatorname{div}_{x}\left(f_{i}(x) \rho_{t}\right) \mathrm{d} \mathbf{W}_{t}^{i} & \text { in } \quad(0, T) \times \mathbb{R}^{n}, \\ \rho_{0}=\mu & \text { on } \quad\{0\} \times \mathbb{R}^{n}\end{cases}
$$


when $\mathbf{W}$ is again a weakly geometric rough path. As before we use the notation $\Gamma_{i}=$ $f_{i}(x) \cdot D_{x}$, whose formal adjoint is $\Gamma_{i}^{\star}=-\operatorname{div}_{x}\left(f_{i} \cdot\right)$.

Definition 4.5 Let $\gamma \in(0,1), \mathbf{W} \in \mathscr{C}_{g}^{\gamma}$ and $\mu \in \mathcal{M}\left(\mathbb{R}^{n}\right)$. Any function $\rho:[0, T] \rightarrow \mathcal{M}\left(\mathbb{R}^{n}\right)$ such that $\rho_{0}=\mu$ is called a weak or measure-valued solution to the rough continuity equation

$$
\mathrm{d} \rho_{t}=\sum_{i=1}^{d} \operatorname{div}_{x}\left(f_{i}(x) \rho_{t}\right) \mathrm{d} \mathbf{W}_{t}^{i}
$$

if for every $\phi$ bounded in $\mathrm{C}_{b}^{N_{\gamma}+1}$ and any word $w$ with $|w| \leq N_{\gamma}$ one has the estimates

$$
\rho_{t}\left(\Gamma_{w} \phi\right) \underset{\left(N_{\gamma}+1-|w|\right) \gamma}{=} \sum_{0 \leq|v|<N_{\gamma}+1-|w|} \rho_{s}\left(\Gamma_{w v} \phi\right)\left\langle\mathbf{W}_{s t}, e_{v}\right\rangle
$$

for every $s<t \in[0, T]$ and uniformly in $\phi$.

Theorem 4.6 Let $f \in \mathcal{C}_{b}^{2 N_{\gamma}+1}$ and $\mathbf{W} \in \mathscr{C}_{g}^{\gamma}$. Given initial data $\mu \in \mathcal{M}\left(\mathbb{R}^{n}\right)$, there exists a unique solution to the measure-valued rough continuity equation, explicitly given for $\phi \in$ $\mathrm{C}_{b}^{N_{\gamma}+1}$ by

$$
\rho_{t}(\phi)=\int \phi\left(X_{t}^{0, x}\right) \mu(d x)
$$

where $X^{0, x}$ is the unique solution of the $R D E \mathrm{~d} X_{t}=\sum_{i=1}^{d} f_{i}\left(X_{t}\right) \mathrm{d} \mathbf{W}_{t}^{i}$ such that $X_{0}^{0, x}=x$.

Proof (Existence) Using the composition of the controlled rough path $\mathbf{X}^{0, x}$ with $\phi \in \mathcal{C}_{b}^{N_{\gamma}+1}$ and the shorthand notation $X_{t}^{0, x}=X_{t}$ we can write

$$
\begin{array}{r}
\phi\left(X_{t}\right) \underset{\left(N_{\gamma}+1\right) \gamma}{=} \phi\left(X_{S}\right)+\sum_{k=1}^{N_{\gamma}} \sum_{|w|=k} \Gamma_{w} \phi\left(X_{S}\right)\left\langle\mathbf{W}_{s t}, w\right\rangle, \\
\Gamma_{i_{1} \ldots i_{n}} \phi\left(X_{t}\right)_{\left(N_{\gamma}+1-n\right) \gamma}^{=} \Gamma_{i_{1} \ldots i_{n}} \phi\left(X_{S}\right)+\sum_{k=1}^{N_{\gamma}-n} \sum_{|w|=k} \Gamma_{i_{1} \ldots i_{n} w} \phi\left(X_{S}\right)\left\langle\mathbf{W}_{s t}, w\right\rangle .
\end{array}
$$

This showing the existence when $\mu=\delta_{x}$ thanks to Proposition 4.3. Since we are dealing with bounded vector fields, all these estimates are uniform in $X_{0}=x$. Thus we can integrate both sides with respect to the measure $\mu$, obtaining the existence.

(Uniqueness) To prove the uniqueness, we will show that for any $0<t \leq T$, any function $g \in \mathcal{C}_{b}^{N_{\gamma}+1}$ and any solution $u:[0, t] \times \mathbb{R}^{n} \rightarrow \mathbb{R}$ of the RPDE

$$
\mathrm{d} u_{r}=\sum_{i=1}^{d} \Gamma_{i} u(r, x) \mathrm{d} \mathbf{W}_{r}^{i}, \quad u_{t}=g,
$$

the function $r \in[0, t] \mapsto \alpha(r):=\rho_{r}\left(u_{r}\right)$ is constant. This property implies that for any function $g \in \mathcal{C}_{b}^{N_{\gamma}+1}$ and $t>0$ one has the identity

$$
\rho_{t}(g)=\rho_{t}\left(u_{t}\right)=\rho_{0}\left(u_{0}\right)=\mu\left(u_{0}\right)
$$

which uniquely determines the measure $\rho_{t}$ for any $0<t \leq T$. Since the parameter $T$ was also arbitrary it is not restrictive to prove the result when $t=T$. Then $\alpha$ is constant if and 
only if one has the estimate

$$
\alpha(r) \underset{\left(N_{\gamma}+1\right) \gamma}{=} \alpha(s) .
$$

Writing $u_{s, r}=u_{r}-u_{s}$ and similarly for $\rho$ one has

$$
\rho_{r}\left(u_{r}\right)-\rho_{s}\left(u_{s}\right)=\rho_{s, r}\left(u_{r}\right)+\rho_{s}\left(u_{s, r}\right) .
$$

By construction of regular solution with $\phi=u_{r} \in \mathrm{C}_{b}^{N_{\gamma}+1}$ the first summand expands as

$$
\rho_{s, r}\left(u_{r}\right) \underset{\left(N_{\gamma}+1\right) \gamma}{=} \sum_{k=1}^{N_{\gamma}} \sum_{|w|=k} \rho_{s}\left(\Gamma_{w} u_{r}\right)\left\langle\mathbf{W}_{s r}, w\right\rangle .
$$

On the other hand, we expand the second summand on the right-hand using the very definition of regular backward RPDE obtaining

$$
u_{s, r}(x) \underset{\left(N_{\gamma}+1\right) \gamma}{=}-\sum_{k=1}^{N_{\gamma}} \sum_{|w|=k} \Gamma_{w} u_{r}(x)\left\langle\mathbf{W}_{s r}, w\right\rangle,
$$

where the remainder is uniform on $x$. By integrating this estimate on $\rho_{s}$, we obtain

$$
\rho_{s}\left(u_{s, r}\right) \underset{\left(N_{\gamma}+1\right) \gamma}{=}-\sum_{k=1}^{N_{\gamma}} \sum_{|w|=k} \rho_{s}\left(\Gamma_{w} u_{r}\right)\left\langle\mathbf{W}_{s r}, w\right\rangle .
$$

Combining the two estimates (4.15) and (4.14) we obtain (4.13) and the theorem is proven.

Acknowledgements CB has received funding from DFG research unit FOR2402, ADj and NT have received funding from Excellence Cluster MATH+ (AA4 and EF1, respectively). PKF has received funding from the European Research Council (ERC) under the European Union's Horizon 2020 research and innovation programme (grant agreement No. 683164).

Funding Open Access funding enabled and organized by Projekt DEAL.

Open Access This article is licensed under a Creative Commons Attribution 4.0 International License, which permits use, sharing, adaptation, distribution and reproduction in any medium or format, as long as you give appropriate credit to the original author(s) and the source, provide a link to the Creative Commons licence, and indicate if changes were made. The images or other third party material in this article are included in the article's Creative Commons licence, unless indicated otherwise in a credit line to the material. If material is not included in the article's Creative Commons licence and your intended use is not permitted by statutory regulation or exceeds the permitted use, you will need to obtain permission directly from the copyright holder. To view a copy of this licence, visit http://creativecommons.org/licenses/by/4.0/.

\section{References}

1. Ambrosio, L.: Transport equation and Cauchy problem for bv vector fields. Invent. Math. 158(2), 227-260 (2004)

2. Beck, L., Flandoli, F., Gubinelli, M., Maurelli, M.: Stochastic ODEs and stochastic linear PDEs with critical drift: regularity, duality and uniqueness. Electron. J. Probab. 24, 72 (2019) (Paper no. 136)

3. Bailleul, I., Gubinelli, M.: Unbounded rough drivers. Ann. Faculté Sci. Toulous. Math. 26(4), 795-830 (2017)

4. Catellier, R.: Rough linear transport equation with an irregular drift. Stoch. Partial Differ. Equations Anal. Comput. 4(3), 477-534 (2016) 
5. Caruana, M., Friz, P.: Partial differential equations driven by rough paths. J. Differ. Equations 247(1), 140-173 (2009)

6. Catellier, R., Gubinelli, M.: Averaging along irregular curves and regularisation of ODEs. Stoch. Process. Appl. 126(8), 2323-2366 (2016)

7. Diehl, J., Friz, P., Stannat, W.: Stochastic partial differential equations: a rough paths view on weak solutions via Feynman-Kac. Ann. Fac. Sci. Toulous. Math. 26(4), 911-947 (2017)

8. Deya, A., Gubinelli, M., Hofmanová, M., Tindel, S.: A priori estimates for rough PDEs with application to rough conservation laws. J. Funct. Anal. 276(12), 3577-3645 (2019)

9. Ronald, J.: DiPerna and Pierre-Louis Lions, ordinary differential equations, transport theory and Sobolev spaces. Invent. Math. 98(3), 511-547 (1989)

10. Flandoli, F., Gubinelli, M., Priola, E.: Well-posedness of the transport equation by stochastic perturbation. Invent. Math. 180(1), 1-53 (2009)

11. Peter, K.: Friz and Martin Hairer. A course on rough paths. Springer, New York (2014)

12. Flandoli, F., Olivera, C.: Well-posedness of the vector advection equations by stochastic perturbation. J. Evol. Equations 18(2), 277-301 (2018)

13. Funaki, T.: Construction of a solution of random transport equation with boundary condition. J. Math. Soc. Jpn. 31(4), 719-744 (1979)

14. Peter, K.: Friz and Nicolas Victoir, A note on the notion of geometric rough paths. Probab. Theory Relat. Fields 136(3), 395-416 (2006)

15. Peter, K.: Friz and Nicolas Victoir, Multidimensional stochastic processes as rough paths, Cambridge Studies in Advanced Mathematics, vol. 120. Cambridge University Press, Cambridge (2010)

16. Gubinelli, M.: Ramification of rough paths. J. Differ. Equations 248(4), 693-721 (2010)

17. Hardy, M.: Combinatorics of partial derivatives. Electron. J. Combin. 13(1), 1-13 (2006) (Research Paper)

18. Hairer, M., Kelly, D.: Geometric versus non-geometric rough paths. Ann. 1'Institut Henri Poincaré Prob. et Stat. 51(1), 207-251 (2015)

19. Lyons, T., Victoir, N.: An extension theorem to rough paths. Ann. Inst. H. Poincaré Anal. Non Linéaire 24(5), 835-847 (2007)

20. Manchon, D.: Hopf algebras in renormalisation, Handbook of algebra. Vol. 5, Handb. Algebr., vol. 5, pp. 365-427. Elsevier/North-Holland, Amsterdam (2008)

21. Maurelli, M.: Wiener chaos and uniqueness for stochastic transport equation. C. R. Math. 349(11), 669$672(2011)$

22. Nualart, D., Tindel, S.: A construction of the rough path above fractional Brownian motion using Volterra's representation. Ann. Probab. 39(3), 1061-1096 (2011)

23. Ogawa, S.: A partial differential equation with the white noise as a coefficient. Probab. Theory Relat. Fields 28(1), 53-71 (1973)

24. Olivera, C., Tudor, C.A.: The density of the solution to the stochastic transport equation with fractional noise. J. Math. Anal. Appl. 431(1), 57-72 (2015)

25. Ree, R.: Lie elements and an algebra associated with shuffles. Ann. Math. 68(2), 210-220 (1958)

26. Unterberger, J.: A renormalized rough path over fractional Brownian motion. Commun. Math. Phys. 320(3), 603-636 (2013) 